\title{
GROWTH OF MAGNETIC FIELDS INDUCED BY TURBULENT MOTIONS
}

\author{
Jungyeon Cho ${ }^{1}$, Ethan T. Vishniac ${ }^{2}$, Andrey BeresnyaK $^{3}$, A. LAZARian ${ }^{3}$, And Dongsu Ryu ${ }^{1}$ \\ ${ }^{1}$ Department of Astronomy and Space Science, Chungnam National University, Daejeon, Korea \\ 2 Department of Physics and Astronomy, McMaster University, Hamilton, Ontario L85 4M1, Canada \\ ${ }^{3}$ Department of Astronomy, University of Wisconsin, Madison, WI 53705, USA \\ Received 2008 February 1; accepted 2008 November 21; published 2009 March 9
}

\begin{abstract}
We present numerical simulations of driven magnetohydrodynamic (MHD) turbulence with weak/moderate imposed magnetic fields. The main goal is to clarify dynamics of magnetic field growth. We also investigate the effects of the imposed magnetic fields on the MHD turbulence, including, as a limit, the case of zero external field. Our findings are as follows. First, when we start off simulations with weak mean magnetic field only (or with small scale random field with zero imposed field), we observe that there is a stage at which magnetic energy density grows linearly with time. Runs with different numerical resolutions and/or different simulation parameters show consistent results for the growth rate at the linear stage. Second, we find that, when the strength of the external field increases, the equilibrium kinetic energy density drops by roughly the product of the rms velocity and the strength of the external field. The equilibrium magnetic energy density rises by roughly the same amount. Third, when the external magnetic field is not very strong (say, less than $\sim 0.2$ times the rms velocity when measured in the units of Alfvén speed), the turbulence at large scales remains statistically isotropic, i.e., there is no apparent global anisotropy of order $B_{0} / v$. We discuss implications of our results on astrophysical fluids.
\end{abstract}

Key words: ISM: general - MHD - turbulence

\section{INTRODUCTION}

Most astrophysical fluids are magnetized. Magnetic field in an astrophysical system can be divided into two components: large-scale regular and small-scale random components. The generation of magnetic field may involve two separate issues: generation of the large-scale regular field (or mean field) and generation of small-scale random field.

The generation or growth of large-scale regular fields is an important topic in astrophysics. However, in this paper we assume fixed large-scale regular fields are already present and we only investigate how small-scale random fields are generated from the imposed large-scale fields. That is, we investigate the growth of magnetic energy in the presence of fixed mean fields. Therefore, the generation/growth of large-scale regular field itself is not a topic of this paper. Readers interested in the topic may refer to mean field dynamo theories (see Moffatt 1978; Parker 1979; Krause \& Radler 1980; Brandenburg \& Subramanian 2005). The type of magnetic energy growth we deal with in this paper is the so-called small-scale turbulence dynamo ${ }^{4}$ the origin of which can be traced back to the papers by Batchelor (1950) and Kazantsev (1968). We also study how properties of magnetohydrodynamic (MHD) turbulence (e.g., energy densities, power spectra, global anisotropy) change as the strength of the mean field changes.

When we introduce a mean field in a turbulent medium, the mean field interacts with turbulent motions. There are two

\footnotetext{
4 In this paper, turbulence dynamo means not growth of mean field itself but generation of random field on scales similar to or smaller than the driving scale of turbulence, which is sometimes referred to as fluctuation dynamo (see, for example, Schekochihin et al. 2007; Subramanian 2008). In all our simulations the strengths of the mean fields do not change with time. In this paper, by 'amplification of magnetic field' or 'growth of magnetic field', we actually mean amplification of fluctuating field(s) on scales similar to or smaller than the driving scale. Therefore, the small-scale turbulence dynamo is different from the mean field dynamo, which deals with growth of mean field itself.
}

distinct types of interaction, depending on the strength of the mean magnetic field. When the large-scale regular magnetic field is weak, turbulent motions stretch the magnetic field lines and, as a result, the magnetic energy density increases. MHD turbulence near the scale of the largest energy containing eddies (i.e., the outer scale or the energy injection scale) will be more or less like ordinary hydrodynamic turbulence with small magnetic back reaction.

MHD turbulence in intracluster medium and intergalactic medium may fall in this type of turbulence. The origin of the seed magnetic fields is still uncertain. However, whatever the origin is, turbulence motions can produce small-scale field through the stretching of the seed fields. In this regime, the growth timescale will be of great importance. If the growth timescale is shorter than the age of the universe in a system, we expect that the system is strongly magnetized in the present time. Otherwise, we expect that the system is weakly magnetized.

On the other hand, when the imposed mean magnetic field is strong in the sense that the turbulent eddy turnover rate at the large scale (i.e., $L / v$ ) is slower than the Alfvénic rate of the same scale (i.e., $\left.L / B_{0}\right),{ }^{5}$ the resulting turbulence can be described through the nonlinear interaction of waves. The turbulence can be either strong, meaning that the cascading happens within one eddy turnover time or weak, meaning that the cascading takes more than one eddy turnover time. A classical study of Iroshnikov (1963) and Kraichnan (1965) presents an example of weak MHD turbulence. This is a hypothetical isotropic MHD turbulence, while we know by now that the actual MHD turbulence is anisotropic (Shebalin et al. 1983; Higdon 1986; Goldreich \& Sridhar 1995; Matthaeus et al. 1998; see also Biskamp 2003 and references therein). An exact analytical treatment of weak MHD turbulence can be found in Galtier et al.

\footnotetext{
5 In what follows we measure the magnetic field in the units of Alfvén velocity $V_{A}$.
} 
(2000). A successful ${ }^{6}$ model of the strong MHD turbulence was presented in Goldreich \& Sridhar (1995).

In this paper we deal with the former case: turbulence with weak imposed magnetic field. In the interstellar medium community, this type of turbulence is called super-Alfvénic turbulence, which is favored by some researchers (see Padoan \& Nordlund 1999; Padoan et al. 2004) as a model of turbulence in molecular clouds. In any case, such turbulence is expected to be present in any system with magnetic field below the equipartition value, which gets subjected to intensive driving. As the turbulence kinetic energy decreases with the scale, we expect the Goldreich-Sridhar (1995) model to be valid at some small scale when the equipartition is reached, while at larger scales we expect hydrodynamic motions to increase the energy of magnetic field.

It is common knowledge that the effects of mean magnetic field have important astrophysical implications. In recent years, interest in the turbulent processes in tangled magnetic field has been growing. Relevant astrophysical problems include thermal diffusion in the intracluster medium (see Chandran \& Cowley 1998; Narayan \& Medvedev 2001; Cho et al. 2003; Lazarian 2006), cosmic ray propagation (see Cassano \& Brunetti 2005; Lazarian \& Beresnyak 2006; Brunetti \& Lazarian 2007) as well as star formation (Padoan et al. 2004; Li \& Nakamura 2004; Vazquez-semadeni et al. 2005).

Cho \& Vishniac (2000a) numerically showed that magnetic energy grows until the magnetic energy density gets comparable to the kinetic energy density (see also Kulsrud \& Anderson 1992; Kulsrud et al. 1997). In this paper, we present more comprehensive studies on the topic. Other aspects of the magnetic field generation also require further studies. For example, Haugen \& Brandenburg (2004) discussed spectral change of MHD turbulence by mean field and showed that imposed magnetic field lowers the spectral magnetic energy in the inertial range. Mac Low (1999) demonstrated that mean magnetic field produces anisotropic structures along the mean field direction in strongly compressible MHD turbulence. Lee et al. (2003) discussed the behavior of energy densities and spectral shapes for three different cases (very weak, weak, and strong mean magnetic field cases) but only for two-dimensional MHD turbulence. They showed the flow character in very weak field classes is similar to that of hydrodynamic turbulence, while the strong field cases show spectra shallower than the hydrodynamic one. Schekochihin et al. (2007) discussed the effects of mean fields for different values of magnetic Prandtl numbers. In all the papers above, the increase of magnetic field energy was noticed. In this paper, we present a comprehensive study on the effect of mean magnetic field on three-dimensional MHD turbulence and turbulence dynamo.

Another issue that requires clarification is the effect of mean magnetic field on the decay of MHD turbulence. Stone et al. (1998) and Mac Low et al. (1998) numerically showed that damping timescales of compressible MHD turbulence are comparable to the large-scale eddy turnover time (see McKee \& Ostriker 2007 for a collection of related results). Incompressible MHD turbulence also decays fast (see Cho et al. 2002). While

\footnotetext{
6 The Goldreich \& Sridhar model was successfully tested in incompressible three-dimensional MHD simulations in Cho \& Vishniac (2000b); Maron \& Goldreich (2001); Cho et al. (2002), as well as in compressible three-dimensional MHD simulations in Cho \& Lazarian (2002, 2003). While the particular points of the model, i.e., the particular slopes of the spectrum are still the subject of debates (see Maron \& Goldreich 2001; Boldyrev 2005, 2006; Beresnyak \& Lazarian 2006; Mason et al. 2006), the cornerstone of the model, which is the critical balance, stays untouched.
}

the earlier works were mostly focused on two extreme limitszero (see, for example, Biskamp \& Muller 2000) and strong mean field limits. In what follows we discuss how the decay timescale changes as the mean field strength changes.

We will first consider the regime of very weak mean field. In this regime, we will mainly investigate the growth rate of magnetic field. Then, we will consider the effect of intermediate mean fields. We will investigate how magnetic and kinetic energy densities, anisotropy, and growth rate of magnetic field change with the increase in the strength of the mean field. In this paper, we deal with incompressible MHD turbulence. We describe our numerical methods in Section 2, present our results for the very weak mean field regime in Section 3 and discuss the effects of the mean field in Section 4. We compare MHD and hydrodynamic turbulences in Section 5 and we give discussion in Section 6. We provide our conclusions in Section 7.

\section{NUMERICAL METHODS}

We have used a pseudospectral code to solve the incompressible MHD equations in a periodic box of size $2 \pi$ :

$$
\begin{gathered}
\frac{\partial \mathbf{v}}{\partial t}=(\nabla \times \mathbf{v}) \times \mathbf{v}-(\nabla \times \mathbf{B}) \times \mathbf{B}+v \nabla^{2} \mathbf{v}+\mathbf{f}+\nabla P^{\prime}, \\
\frac{\partial \mathbf{B}}{\partial t}=\nabla \times(\mathbf{v} \times \mathbf{B})+\eta \nabla^{2} \mathbf{B}, \\
\nabla \cdot \mathbf{v}=\nabla \cdot \mathbf{B}=0,
\end{gathered}
$$

where $\mathbf{f}$ is random driving force, $P^{\prime} \equiv P+\mathbf{v} \cdot \mathbf{v} / 2, \mathbf{v}$ is the velocity, and $\mathbf{B}$ is magnetic field divided by $(4 \pi \rho)^{1 / 2}$. We use 21 forcing components with $2 \leqslant k \leqslant \sqrt{12}$. Each forcing component has a correlation time of one. The peak of energy injection occurs at $k \approx 2.5$. The amplitudes of the forcing components are tuned to ensure $v \approx 1$ for the hydrodynamic simulation with $v=0.0074$. Therefore, one eddy turnover time, $\sim L / v$, is approximately 2.5 time units. In this representation, $\mathbf{v}$ can be viewed as the velocity measured in units of the rms velocity, $\mathrm{v}$, of the system and $\mathbf{B}$ as the Alfvén speed in the same units. Other variables have their usual meaning. The magnetic field consists of the uniform background field and the fluctuating field: $\mathbf{B}=\mathbf{B}_{0}+\mathbf{b}$. The Alfvén velocity of the background field, $B_{0}$, varies from 0 to 1 . Throughout the paper, we consider only cases where viscosity is equal to magnetic diffusion: ${ }^{7}$

$$
v=\eta \text {. }
$$

In pseudospectral methods, we calculate the temporal evolution of the Equations (1) and (2) in Fourier space. To obtain the Fourier components of nonlinear terms, we first calculate them in real space, and transform back into Fourier space. We use exactly the same forcing terms for all simulations. The average kinetic helicity in these simulations is negative.

\footnotetext{
In this paper, our goal is to study small-scale turbulence dynamo properties in the asymptotic limit of very small viscosity and very small magnetic diffusion. Unit magnetic Prandtl number $(\nu=\eta)$ will be a good approximation in this limit. To support this claim, we ran a simulation that is very similar to $256 \mathrm{H} 3-B_{0} 10^{-3}$ but the magnetic Prandtl number $(=v / \eta)$ is 0.01 . The qualitative behavior of time evolution (not shown in this paper) is similar to that of the unit magnetic Prandtl number case: magnetic energy density grows initially and saturates later when magnetic energy density becomes comparable to the kinetic one. The average values of $v^{2}$ and $b^{2}$ are $\sim 0.78$ and $\sim 0.25$, respectively. Average is taken over the time interval of $(50,110)$. The magnetic energy density is smaller than that of $256 \mathrm{H} 3-B_{0} 10^{-3}$ (see Table 1) because magnetic dissipation occurs at smaller wavenumbers.
} 
We use an appropriate projection operator to calculate $\nabla P^{\prime}$ term in Fourier space and also to enforce divergence-free condition $(\nabla \cdot \mathbf{v}=\nabla \cdot \mathbf{B}=0)$. We use up to $384^{3}$ collocation points. At $t=0$, the magnetic field has either only uniform component (when $B_{0} \neq 0$ ) or only random components (when $B_{0}=0$ ) and the velocity has a support between $2 \leqslant k \leqslant 4$ in wavevector space.

Either physical viscosity (and diffusion) or hyperviscosity (and hyperdiffusion) is used for dissipation terms (see Table 1). The power of hyperviscosity is set to 3 or 8 , such that the dissipation term in the above equation is replaced with

$$
-v_{n}\left(\nabla^{2}\right)^{n} \mathbf{v}
$$

where $n=3$ or 8 and $v_{n}$ is determined from the condition $v_{n}(N / 2)^{2 n} \Delta t \approx 0.5{ }^{8}$ Here $\Delta t$ is the time step and $N$ is the number of grid points in each direction. The same expression is used for the magnetic dissipation term. We list parameters used for the simulations in Table 1 . We use the notation $X Y-B_{0} Z$, where $X=384,320,256,144,96$, or 64 refers to the number of grid points in each spatial direction; $Y=H$ or $P$ refers to hyperor physical viscosity; $\mathrm{Z}$ refers to the strength of the external magnetic fields. Diagnostics of our code can be found in Cho \& Vishniac (2000a).

We use the following notations.

1. $B=\sqrt{B_{0}^{2}+b^{2}}$ : total magnetic field strength or its Alfvén speed.

2. $B_{0}$ : mean magnetic field or its Alfvén speed.

3. $b, v\left(=v_{\mathrm{rms}}\right)$ : the average rms random magnetic field and velocity. Average is taken after turbulence reaches a statistically stationary state.

4. $b^{(0)}, v^{(0)}$ : the zeroth-order magnetic field and velocity when $B_{0}=0$.

5. $E_{v}(k): E_{v}(k)=(1 / 2) \sum_{k-0.5 \leqslant k^{\prime}<k+0.5}\left|\hat{\mathbf{v}}_{\mathbf{k}^{\prime}}\right|^{2}$, where $\hat{\mathbf{v}}_{\mathbf{k}^{\prime}}$ is the Fourier component of velocity. We define the magnetic energy spectrum $E_{b}(k)$ similarly.

\section{SMALL-SCALE TURBULENCE DYNAMO IN THE VERY WEAK MEAN FIELD LIMIT}

\subsection{Expectations}

One of the most important issues in this regime is the generation of small-scale random fields from the large-scale regular fields. Since large-scale regular magnetic fields are observed in almost all astrophysical objects, this issue is of great importance in astrophysics. Cho \& Vishniac (2000a; see also Kulsrud \& Anderson 1992; Kulsrud et al. 1997) argued that magnetic energy in this regime grows through field line stretching and that there are two stages of magnetic field amplification. During the first stage, stretching is most active near, or somewhat larger than, the dissipation scale (spectral cutoff scale) and the magnetic energy spectrum peaks at this scale. It is clear that magnetic energy grows exponentially during this stage and that the characteristic timescale is the eddy turnover time at the dissipation scale. As the magnetic energy grows, the magnetic back reaction becomes important at the dissipation scale. When energy equipartition between kinetic energy and

\footnotetext{
8 When a high-order hyperdiffusion is used, the spectral properties near the dissipation cutoff are affected by a strong bottleneck effect. The bottleneck effect affects high- $k$ Fourier components. Since we study mostly the behavior of the total $v^{2}$ and $b^{2}$ which depends mostly on small- $k$ Fourier components, we believe the bottleneck effect is not a serious issue in our study.
}

magnetic energy is reached at this scale, the stretching rate slows down and a second stage of slower growth begins. Figure 3 of Cho \& Vishniac (2000a) shows that during this stage the peak of the magnetic power spectrum moves to larger scales. Using phenomenological arguments similar to those above, Shekochihin \& Cowley (2007) argued that magnetic energy grows linearly during the second stage,

$$
\frac{d B^{2}}{d t} \sim \epsilon
$$

where $\epsilon$ is the energy injection rate, which should be equal to the total energy dissipation rate in a statistically stationary state. The linear stage of magnetic energy growth ends when stretching on the energy injection scale becomes suppressed, which occurs when the magnetic energy density becomes comparable to the kinetic energy density.

\subsection{Growth Rate at the Linear Stage}

Let us first consider the growth rate of magnetic energy during the linear stage, which has an important consequence for the strength of the magnetic fields in the large-scale structure of the universe (see Ryu et al. 2008). Figure 1 shows time evolution of $v^{2}$ and $b^{2}$. All simulations started with mean magnetic field strength of $B_{0}=0.001$. No random magnetic component was present at the beginning of the simulation. As simulations go on, random magnetic components are generated and magnetic energy grows through stretching of magnetic field lines. The growth of magnetic energy is slow when viscosity (and magnetic diffusivity) is high. For example, Run $64 \mathrm{P} 1-B_{0} 10^{-3}$ shows substantially slower growth rate than Run $256 \mathrm{P}-B_{0} 10^{-3}$. The growth rate seems to show a convergence as viscosity decreases. For example, there is no big difference in magnetic field growth rate between Run $256 \mathrm{P}-B_{0} 10^{-3}$ and Run $256 \mathrm{H} 3-B_{0} 10^{-3}$.

We compare the growth rates using simulations with different parameters. Right panel of Figure 1 shows that the magnetic energy growth rates during the linear growth stage are very similar. Note that we use proper normalization for both horizontal and vertical axes. We plot only high-resolution runs. Runs with physical viscosity (and magnetic diffusion) show slightly smaller slopes, which is reasonable. The strength of mean magnetic field does not seem to affect the linear growth rate. However, we can clearly observe that, when the mean field is weaker, the onset of the linear stage occurs later. It is also worth noting that even the run with zero mean field (256H8$\left.B_{0} 0\right)$ shows a similar linear growth rate. In the run with zero mean field $\left(256 \mathrm{H} 8-B_{0} 0\right)$, only a small-scale $(k \sim 70)$ random magnetic field is present at $t=0$.

In code units, the linear growth stage ends at $t \sim 40$. The values of $B^{2}$ at that time is $\sim 0.4$. Therefore, the slope during the linear growth stage is around 0.01 . But, when we represent the slope in terms of normalized energy density and time, we obtain different slopes:

$$
\frac{B^{2}(t)}{2 E_{\mathrm{turb}}} \sim 0.07 \frac{\epsilon}{2 E_{\mathrm{turb}}(v / L)} \frac{t}{L / v}+\text { const. }
$$

or

$$
\frac{B^{2}(t)}{2 E_{\text {turb }}} \sim 0.033 \frac{t}{L / \sqrt{v^{2}+b^{2}}}+\text { const. }
$$


Table 1

Results of Simulations

\begin{tabular}{|c|c|c|c|c|c|c|c|c|}
\hline Run $^{a}$ & $N^{3}$ & $v=\eta$ & $B_{0}^{2}$ & $v^{2}$ & $b^{2 \mathrm{~b}}$ & $\epsilon$ & $D_{M}$ & $\left(t_{1}, t_{2}\right)^{\mathrm{c}}$ \\
\hline $320 \mathrm{P}-B_{0} 10^{-3}$ & $320^{3}$ & 0.0008 & $10^{-6}$ & 0.662 & 0.283 & 0.157 & 0.110 & $(60,122)$ \\
\hline $256 \mathrm{H} 8-B_{0} 0$ & $256^{3}$ & hyper & 0 & 0.616 & 0.455 & $\ldots$ & $\ldots$ & $(60,150)$ \\
\hline $256 \mathrm{H} 8-B_{0} 10^{-6}$ & $256^{3}$ & hyper & $10^{-12}$ & 0.601 & 0.432 & $\ldots$ & $\ldots$ & $(60,150)$ \\
\hline $256 \mathrm{H} 8-B_{0} 10^{-3}$ & $256^{3}$ & hyper & $10^{-6}$ & 0.592 & 0.443 & $\ldots$ & $\ldots$ & $(60,150)$ \\
\hline $256 \mathrm{H} 8-B_{0} 10^{-1}$ & $256^{3}$ & hyper & $10^{-2}$ & 0.495 & 0.566 & $\ldots$ & $\ldots$ & $(30,131)$ \\
\hline $256 \mathrm{H} 3-B_{0} 10^{-3}$ & $256^{3}$ & hyper & $10^{-6}$ & 0.613 & 0.404 & $\ldots$ & $\ldots$ & $(60,150)$ \\
\hline $256 \mathrm{H} 3-B_{0} 10^{-2}$ & $256^{3}$ & hyper & $10^{-4}$ & 0.633 & 0.388 & $\ldots$ & $\ldots$ & $(60,150)$ \\
\hline $256 \mathrm{H} 3-B_{0} 10^{-1.5}$ & $256^{3}$ & hyper & $10^{-3}$ & 0.606 & 0.417 & $\ldots$ & $\ldots$ & $(60,150)$ \\
\hline $256 \mathrm{H} 3-B_{0} 10^{-1}$ & $256^{3}$ & hyper & $10^{-2}$ & 0.504 & 0.508 & $\ldots$ & $\ldots$ & $(30,90)$ \\
\hline $256 \mathrm{H} 3-B_{0} 6^{-1}$ & $256^{3}$ & hyper & $1 / 36$ & 0.453 & 0.584 & $\ldots$ & $\ldots$ & $(30,46)$ \\
\hline $256 \mathrm{H} 3-B_{0} 10^{-0.5}$ & $256^{3}$ & hyper & $1 / 10$ & 0.423 & 0.618 & $\ldots$ & $\cdots$ & $(30,43)$ \\
\hline $256 \mathrm{H} 3-B_{0} 1$ & $256^{3}$ & hyper & 1 & 0.603 & 0.449 & $\ldots$ & $\ldots$ & $(15,45)$ \\
\hline $256 \mathrm{P}-B_{0} 10^{-3}$ & $256^{3}$ & 0.001 & $10^{-6}$ & 0.711 & 0.250 & 0.159 & 0.109 & $(60,150)$ \\
\hline $256 \mathrm{P}-B_{0} 10^{-2}$ & $256^{3}$ & 0.001 & $10^{-4}$ & 0.672 & 0.275 & 0.158 & 0.110 & $(60,135)$ \\
\hline $256 \mathrm{P}-B_{0} 10^{-1.5}$ & $256^{3}$ & 0.001 & $10^{-3}$ & 0.639 & 0.285 & 0.155 & 0.107 & $(60,120)$ \\
\hline $256 \mathrm{P}-B_{0} 10^{-1}$ & $256^{3}$ & 0.001 & $10^{-2}$ & 0.559 & 0.370 & 0.157 & 0.108 & $(30,60)$ \\
\hline $256 \mathrm{P}-B_{0} 5^{-1}$ & $256^{3}$ & 0.001 & $1 / 25$ & 0.467 & 0.460 & 0.145 & 0.0970 & $(30,90)$ \\
\hline $256 \mathrm{P}-B_{0} 10^{-0.5}$ & $256^{3}$ & 0.001 & $1 / 10$ & 0.437 & 0.500 & 0.136 & 0.0884 & $(30,90)$ \\
\hline $256 \mathrm{P}-B_{0} 0.8$ & $256^{3}$ & 0.001 & 0.64 & 0.512 & 0.458 & 0.109 & 0.0609 & $(15,45)$ \\
\hline $256 \mathrm{P}-B_{0} 1$ & $256^{3}$ & 0.001 & 1 & 0.599 & 0.403 & 0.101 & 0.0540 & $(15,34)$ \\
\hline $144 \mathrm{H} 8-B_{0} 10^{-3.5}$ & $144^{3}$ & hyper & $10^{-7}$ & 0.649 & 0.420 & 0.161 & $\ldots$ & $(60,240)$ \\
\hline $144 \mathrm{H} 8-B_{0} 10^{-1.5}$ & $144^{3}$ & hyper & $10^{-3}$ & 0.632 & 0.438 & 0.163 & $\ldots$ & $(60,220)$ \\
\hline $144 \mathrm{H} 8-B_{0} 16^{-1}$ & $144^{3}$ & hyper & $1 / 256$ & 0.576 & 0.487 & 0.158 & $\cdots$ & $(60,220)$ \\
\hline $144 \mathrm{H} 8-B_{0} 10^{-1}$ & $144^{3}$ & hyper & $1 / 100$ & 0.526 & 0.515 & 0.150 & $\ldots$ & $(30,90)$ \\
\hline $144 \mathrm{H} 8-B_{0} 10^{-0.5}$ & $144^{3}$ & hyper & $1 / 10$ & 0.458 & 0.613 & 0.133 & $\ldots$ & $(30,90)$ \\
\hline $144 \mathrm{P}-B_{0} 10^{-3}$ & $144^{3}$ & 0.0025 & $10^{-6}$ & 0.728 & 0.214 & 0.165 & 0.102 & $(60,350)$ \\
\hline $144 \mathrm{P}-B_{0} 10^{-2}$ & $144^{3}$ & 0.0025 & $10^{-4}$ & 0.743 & 0.210 & 0.166 & 0.102 & $(70,350)$ \\
\hline $144 \mathrm{P}-B_{0} 10^{-1.5}$ & $144^{3}$ & 0.0025 & $10^{-3}$ & 0.709 & 0.227 & 0.164 & 0.103 & $(70,350)$ \\
\hline $144 \mathrm{P}-B_{0} 10^{-1}$ & $144^{3}$ & 0.0025 & $1 / 100$ & 0.589 & 0.312 & 0.162 & 0.107 & $(70,350)$ \\
\hline $144 \mathrm{P}-B_{0} 10^{-0.5}$ & $144^{3}$ & 0.0025 & $1 / 10$ & 0.450 & 0.453 & 0.147 & 0.095 & $(70,350)$ \\
\hline $144 \mathrm{P}-B_{0} 1$ & $144^{3}$ & 0.0025 & 1 & 0.570 & 0.346 & 0.092 & 0.045 & $(70,350)$ \\
\hline $96 \mathrm{P}-B_{0} 0$ & $96^{3}$ & 0.0043 & 0 & 0.766 & 0.165 & 0.164 & 0.086 & $(200,500)$ \\
\hline $96 \mathrm{P}-B_{0} 10^{-3.5}$ & $96^{3}$ & 0.0043 & $10^{-7}$ & 0.761 & 0.172 & 0.166 & 0.088 & $(200,500)$ \\
\hline $96 \mathrm{P}-B_{0} 10^{-2}$ & $96^{3}$ & 0.0043 & $10^{-4}$ & 0.765 & 0.168 & 0.169 & 0.090 & $(200,500)$ \\
\hline $96 \mathrm{P}-B_{0} 10^{-1}$ & $96^{3}$ & 0.0043 & $1 / 100$ & 0.608 & 0.268 & 0.164 & 0.100 & $(200,400)$ \\
\hline $96 \mathrm{P}-B_{0} 10^{-0.5}$ & $96^{3}$ & 0.0043 & $1 / 10$ & 0.447 & 0.404 & 0.149 & 0.095 & $(200,400)$ \\
\hline $64 \mathrm{P} 1-B_{0} 0$ & $64^{3}$ & 0.0074 & 0 & 0.784 & 0.115 & 0.165 & 0.064 & $(300,800)$ \\
\hline $64 \mathrm{P} 1-B_{0} 10^{-3.5}$ & $64^{3}$ & 0.0074 & $10^{-7}$ & 0.786 & 0.113 & 0.166 & .064 & $(300,800)$ \\
\hline $64 \mathrm{P} 1-B_{0} 10^{-2}$ & $64^{3}$ & 0.0074 & $10^{-4}$ & 0.778 & 0.119 & 0.166 & .066 & $(300,800)$ \\
\hline $64 \mathrm{P} 1-B_{0} 10^{-1.5}$ & $64^{3}$ & 0.0074 & $10^{-3}$ & 0.743 & 0.137 & 0.164 & 0.071 & $(300,800)$ \\
\hline $64 \mathrm{P} 1-B_{0} 10^{-1}$ & $64^{3}$ & 0.0074 & $1 / 100$ & 0.617 & 0.221 & 0.166 & 0.089 & $(300,800)$ \\
\hline $64 \mathrm{P} 1-B_{0} 10^{-0.5}$ & $64^{3}$ & 0.0074 & $1 / 10$ & 0.441 & 0.350 & 0.153 & 0.093 & $(300,500)$ \\
\hline $64 \mathrm{P} 1-B_{0} 1$ & $64^{3}$ & 0.0074 & 1 & 0.519 & 0.278 & 0.096 & 0.044 & $(300,800)$ \\
\hline $64 \mathrm{P} 2-B_{0} 0$ & $64^{3}$ & 0.015 & 0 & 0.806 & 0.0215 & 0.164 & 0.014 & $(300,750)$ \\
\hline $64 \mathrm{P} 2-B_{0} 10^{-3.5}$ & $64^{3}$ & 0.015 & $10^{-7}$ & 0.808 & 0.0201 & 0.164 & 0.013 & $(300,750)$ \\
\hline $64 \mathrm{P} 2-B_{0} 10^{-2}$ & $64^{3}$ & 0.015 & $10^{-4}$ & 0.781 & 0.0364 & 0.165 & 0.022 & $(300,750)$ \\
\hline $64 \mathrm{P} 2-B_{0} 10^{-1.5}$ & $64^{3}$ & 0.015 & $10^{-3}$ & 0.730 & 0.0660 & 0.166 & 0.037 & $(300,750)$ \\
\hline $64 \mathrm{P} 2-B_{0} 10^{-1}$ & $64^{3}$ & 0.015 & $1 / 100$ & 0.592 & 0.145 & 0.166 & 0.065 & $(300,750)$ \\
\hline $64 \mathrm{P} 2-B_{0} 10^{-0.5}$ & $64^{3}$ & 0.015 & $1 / 10$ & 0.413 & 0.269 & 0.155 & 0.085 & $(300,750)$ \\
\hline $64 \mathrm{P} 2-B_{0} 1$ & $64^{3}$ & 0.015 & 1 & 0.409 & 0.210 & 0.095 & 0.041 & $(300,750)$ \\
\hline 256H8-hydro & $256^{3}$ & hyper & $\ldots$ & 1.225 & $\ldots$ & 0.168 & $\ldots$ & $(15,30)$ \\
\hline 256H3-hydro & $256^{3}$ & hyper & $\ldots$ & 1.207 & $\ldots$ & 0.165 & $\ldots$ & $(15,28)$ \\
\hline 256P-hydro & $256^{3}$ & 0.001 & $\ldots$ & 1.080 & $\ldots$ & 0.150 & $\ldots$ & $(15,75)$ \\
\hline 144H8-hydro & $144^{3}$ & hyper & $\ldots$ & 1.118 & $\ldots$ & $\ldots$ & $\ldots$ & $(8,18)$ \\
\hline 144P-hydro & $144^{3}$ & 0.0025 & $\ldots$ & 1.090 & $\ldots$ & 0.164 & $\ldots$ & $(60,350)$ \\
\hline 96P-hydro & $96^{3}$ & 0.0043 & $\ldots$ & 1.053 & $\ldots$ & 0.160 & $\ldots$ & $(50,165)$ \\
\hline 64P1-hydro & $64^{3}$ & 0.0074 & $\ldots$ & 0.994 & $\ldots$ & 0.165 & $\ldots$ & $(300,800)$ \\
\hline 64P2-hydro & $64^{3}$ & 0.015 & $\ldots$ & 0.850 & $\ldots$ & 0.163 & $\ldots$ & $(300,750)$ \\
\hline
\end{tabular}

Notes.

${ }^{a}$ Only selected runs are shown in this table.

b $B^{2}=B_{0}^{2}+b^{2}$.

c Time interval used for averaging physical quantities. 

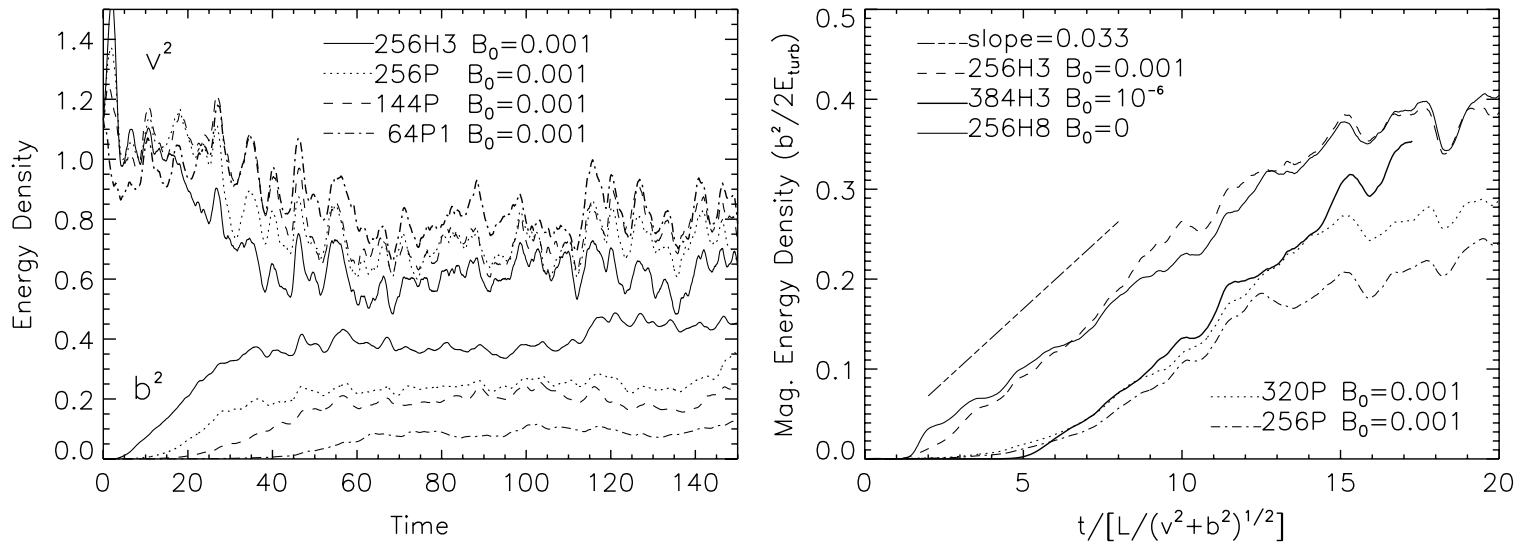

Figure 1. Time evolution of kinetic and magnetic energy densities. Left panel: the level of magnetic energy at the saturation level strongly depends on the value of magnetic diffusivity (= viscosity). Right panel: comparison of magnetic energy growth rates. The growth rates at the linear growth stage are similar. In the case of $256 \mathrm{H} 8-B_{0} 0$, the mean field is zero and the magnetic energy spectrum at $t=0$ peaks near $k \sim 70$. In all other cases, only a weak mean field is present at $t=0$. Note that runs $256 \mathrm{H} 8-B_{0} 0$ (i.e., run with no imposed field) and $256 \mathrm{H} 8-B_{0} 10^{-3}$ show similar growth rates and also similar final saturation levels.

where we use $\epsilon \sim 0.16, L \sim 2.5, v \sim 0.9, v^{2}+B^{2} \sim 1.0$, and $E_{\text {turb }}=\left(v^{2}+B^{2}\right) / 2 \sim 0.5$ (see right panel of Figure 1). ${ }^{9}$ A similar linear growth rate has been observed in a recent work by Ryu et al. (2008), in which they derived strength of magnetic fields in the large-scale structure of the universe. In their model, the linear growth rate derived from a simulation plays an essential role.

\subsection{Saturation Level}

Using data with relatively low numerical resolutions, Cho \& Vishniac (2000a) showed that, in the limit of $v(=\eta) \rightarrow 0$, the magnetic energy density in the saturation stage is comparable to the kinetic energy density. This is consistent with the fact that magnetic fields are amplified through field line stretching: as we mentioned before, stretching of magnetic field at the energy injection scale is suppressed only when the magnetic energy density becomes comparable to the kinetic energy density. In this subsection we present results with higher numerical resolutions.

We list the energy densities in the saturation stage in Table 1. We obtained $v^{2}, b^{2}, \epsilon$, and $D_{M}$ by averaging over $\left(t_{1}, t_{2}\right)$. Here $D_{M}$ is the magnetic energy dissipation rate. It is important to note that these time averages are taken after the turbulence has reached a stationary state. Conclusions based on these averaged values do not apply to the initial growth phase of the magnetic field.

Figure 2 shows $v^{2}$ and $b^{2}$ as functions of $v(=\eta) .{ }^{10}$ All the simulations shown in the left panel of Figure 1 have similar kinetic energy densities. However, the magnetic energy density obviously depends on the Ohmic diffusivity $\eta$. When $v(=\eta)$ goes to zero, the magnetic energy seems to approach $\sim 40 \%$ of the total energy. This is somewhat larger than the value obtained by Haugen et al. (2003), where $\sim 30 \%$ of the total energy is magnetic. The discrepancy may stem from the fact that we use hyperviscosity to achieve a very small viscosity (and magnetic diffusion).

Runs shown in the left panel of Figure 3 show that the order of hyperdiffusion does not strongly affect the growth rate and

\footnotetext{
9 A careful examination of Figure 1 reveals that, although $v^{2}$ decreases and $b^{2}$ increases during the growth stage of magnetic energy, the sum of $v^{2}$ and $b^{2}$ does not change much. In hyperviscosity runs, the value of $v^{2}+b^{2}$ is around 1 all the time. This is why we use $\sqrt{v^{2}+b^{2}}\left(\approx \sqrt{v^{2}+B^{2}}\right)$ in Equation (8).

${ }^{10}$ The use of $\sqrt{v}(=\sqrt{\eta})$ for horizontal axis is not motivated by theoretical considerations, but by clarity of presentations.
}

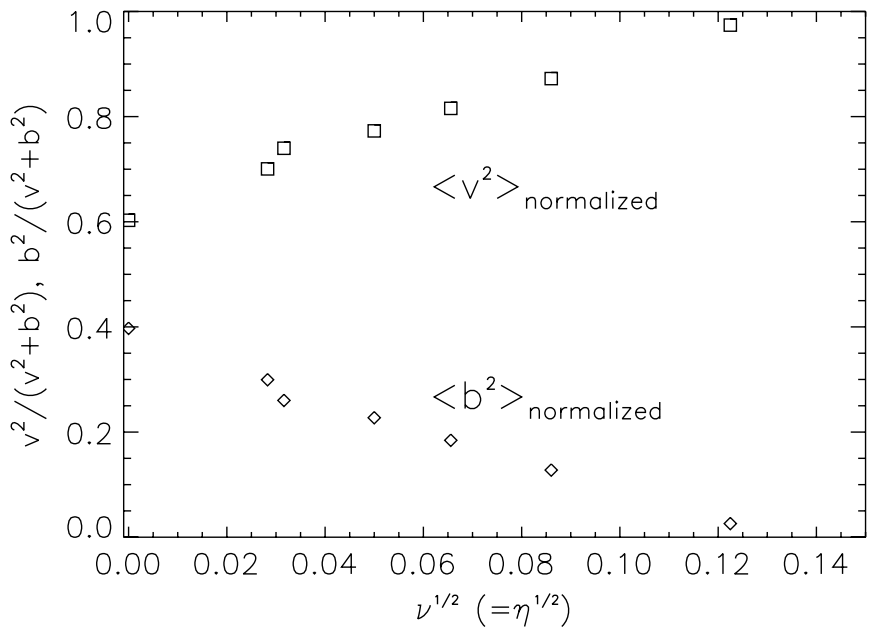

Figure 2. Normalized average kinetic and magnetic energy densities. Average is taken after turbulence has reached a saturation state.

saturation level of magnetic energy. Runs shown in the right panel of Figure 3 show that numerical resolution slightly affects the saturation level of magnetic energy.

\subsection{Exponential Growth Stage}

Magnetic field is amplified through field line stretching, which is initially most active near the dissipation scale. As a result, magnetic energy will grow exponentially at the beginning. To see this stage more clearly, we plot time evolution of magnetic energy in logarithmic scales. We use Run 384H3$B_{0} 10^{-6}$. As expected, Figure 4 clearly shows this exponential growth stage. The strength of the mean field is $10^{-6}$ and the dissipation scale is around $k \sim 100$. When $t<15$, the growth rate is exponential and magnetic energy spectrum peaks near $k \sim 100$. At $t \sim 15$, energy equipartition is reached at $k \sim 100$ and the exponential growth stage ends. After $t \sim 15$, the linear growth stage begins and the peak of magnetic energy spectrum moves to smaller $k$ s.

\section{EFFECTS OF THE MEAN FIELD $\left(B_{0}\right)$}

In this section, we consider a similar numerical setup as in the previous section: only a mean magnetic field $B_{0}$ is present at $t=0$. Then, turbulent motions generate fluctuating magnetic 

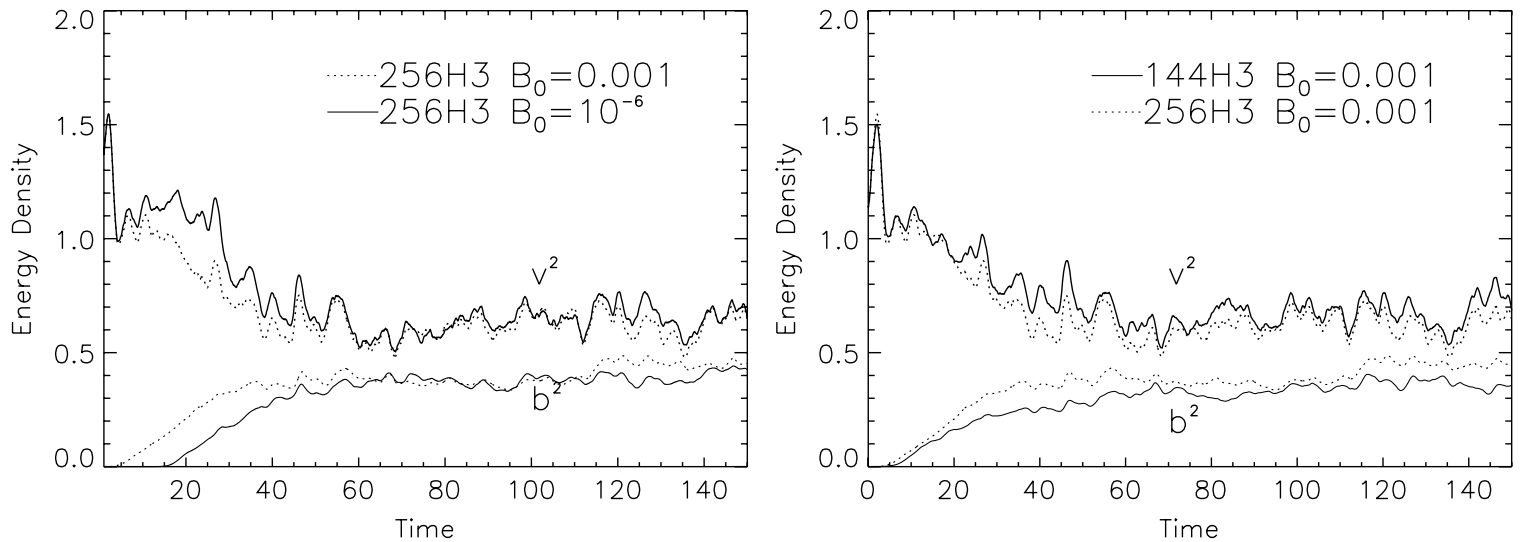

Figure 3. Comparison of runs. Left panel: these runs with hyperdiffusion shows that initial strength of the mean magnetic field does not strongly affect the growth rate and saturation level of magnetic field. However, amplification of magnetic field (in fact, onset of linear growth stage) is delayed when the seed mean magnetic field is weak. The delay will be negligible when the dissipation scale is very small compared with the energy injection scale (see Section 6.3 for details). Right panel: these two runs with hyperdiffusion show that numerical resolution also does not strongly affect the growth timescale, although two runs show slightly different levels of energy saturation.
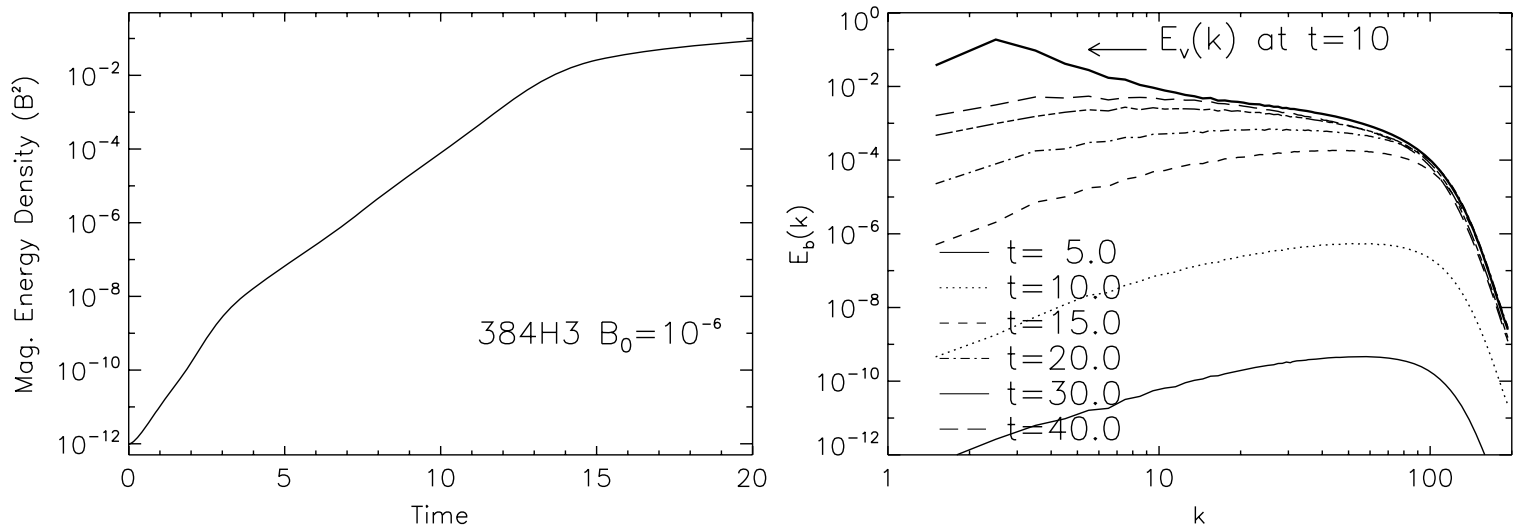

Figure 4. Time evolution of Run $384 \mathrm{H} 3-B_{0} 10^{-6}$. This run with hyperdiffusion shows rapid exponential growth at the beginning, which is due to the stretching of magnetic field lines near the dissipation cut off at $k_{d} \sim 100$. During this phase, magnetic spectra peak at $k \sim 80$ and move upward. After $t \sim 15$, magnetic energy becomes comparable to the kinetic energy at $k_{d} \sim 100$ and the initial rapid exponential growth phase ends.

field $\mathbf{b}$ and, as time goes on, the strength of the fluctuating field grows. After a certain amount of time, which depends on $\eta$ and $B_{0}$, turbulence reaches a statistically stationary state. In this section, we study how the strength of the mean field $\left(B_{0}\right)$ affects energy densities and other properties of turbulence. As we will see in this section, when the mean field is stronger, magnetic energy rises faster, the system reaches the statistically stationary state more quickly, and magnetic energy density at the saturation stage is higher, while kinetic energy density is smaller. This result is consistent with an earlier result by Tao et al. (1993). Although their main conclusion is the suppression of $\alpha$ effect in the presence of mean fields, their figures show that magnetic energy density at the saturation stage is higher when the mean field is stronger. Our result is also consistent with those by Schekochihin et al. (2007), where they studied the effects of mean fields for different magnetic Prandtl numbers.

\subsection{Scaling of Energy Densities, $v^{2}$ and $b^{2}$}

Figure 5 shows time evolution of fluctuating magnetic energy density. When the mean field gets stronger, the saturation level increases and the growth time becomes shorter. Runs with both physical and hyperviscosity show similar behavior. In Figure 6, we explicitly measure the growth timescale, which we define the time the energy of the random magnetic field reaches $80 \%$ of its saturation value. Roughly speaking, Figure 6 implies that the growth timescales with $\sqrt{B_{0}}$. Numerical resolution or the form of viscosity is not important unless the mean field is extremely small.

As we can see in Figure 7, all our simulations, except runs with $B_{0} \sim 1$, have similar energy dissipation/injection rates $\epsilon(=\mathbf{f} \cdot \mathbf{v})$. When the mean magnetic field is very weak, the rate is $\sim 0.16$ and only weakly depends on $v(=\eta)$. The rate declines slowly as the external field gets stronger. We list the total energy dissipation rate and the magnetic energy dissipation rate in Table 1. In $256 \mathrm{P}$ runs, the ratio $D_{M} / \epsilon$ is $\sim 0.7$ in weak mean field cases and it drops to $\sim 0.55$ in strong mean field cases (e.g., $256 \mathrm{P}-B_{0} 0.8$ and $256 \mathrm{P}-B_{01}$ ). The result for weak mean field cases is consistent with Haugen et al. (2004).

We measure average energy densities at the statistically stationary state and list them in Table 1. In Figure 8, we plot the fluctuating energy densities, $v^{2}$ and $b^{2}\left(=B^{2}-B_{0}^{2}\right)$, as functions of mean field strength $B_{0}$.

First, from Figure 8 (and Table 1), we note that there is no difference between turbulence with no external magnetic field and one with very weak external fields. (For example, compare $256 \mathrm{H} 8-B_{0} 0$ and $256 \mathrm{H} 8-B_{0} 10^{-6}$, and $96 \mathrm{P}-B_{0} 0$ and $96 \mathrm{P}-B_{0} 10^{-3.5}$.) When the external fields are very weak, the kinetic and magnetic energy densities go smoothly to the zero external field limit. From the figure, we also note that the kinetic energy densities are not very sensitive to the value of $v(=\eta)$ 

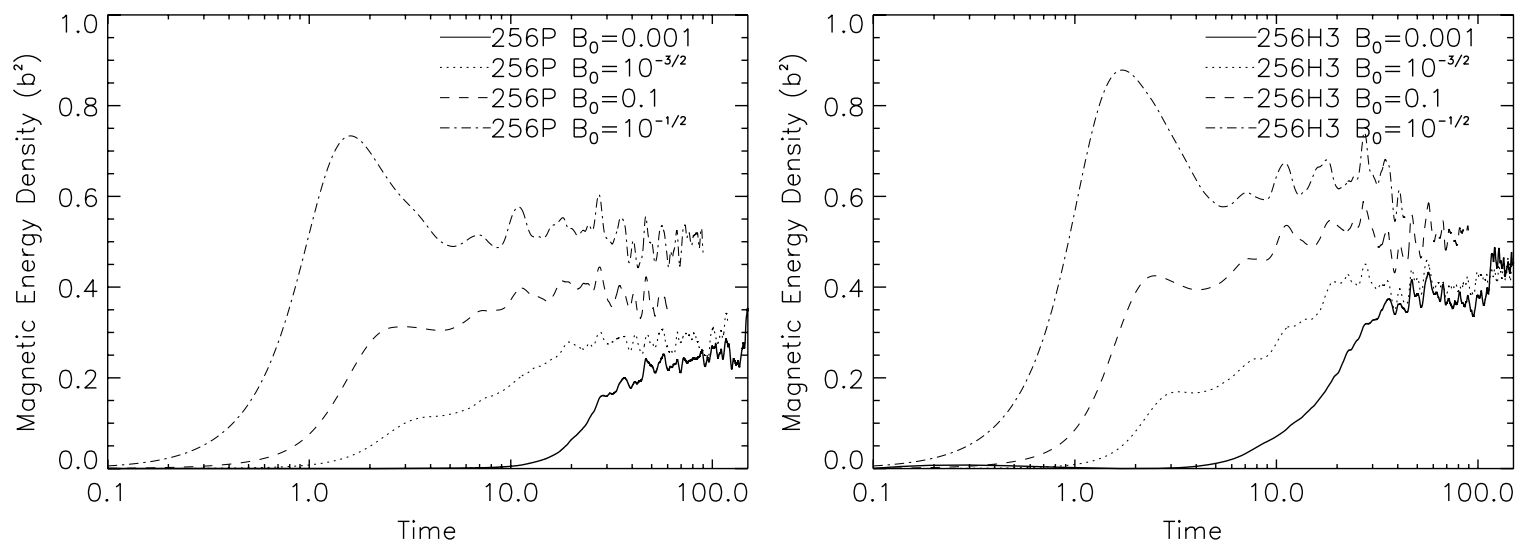

Figure 5. Time evolution of magnetic energy density. The stronger the mean magnetic field, the higher the magnetic energy at the saturation stage. The growth time is shorter when the mean magnetic field is stronger. Left panel: runs with physical diffusion. Right panel: runs with hyperdiffusion.

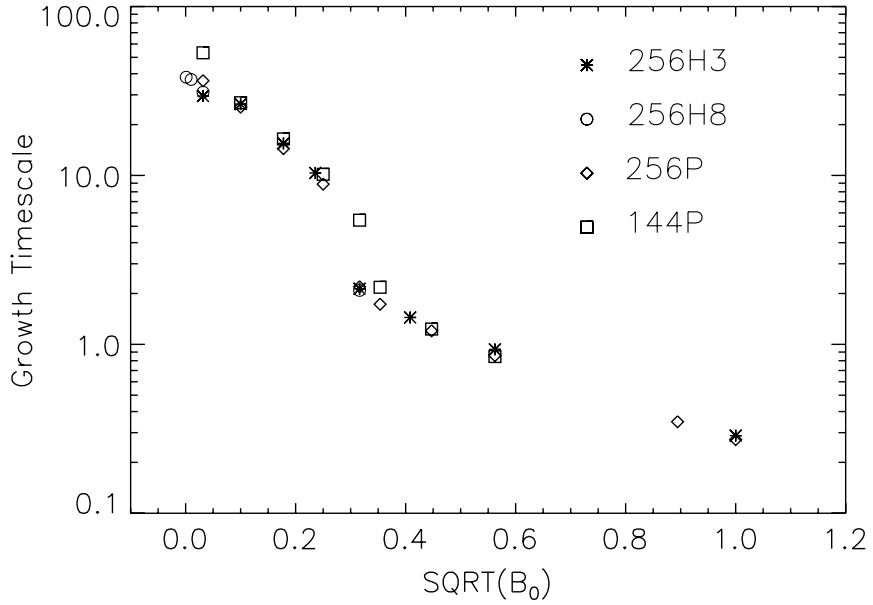

Figure 6. Growth timescale. The $y$-axis is the time in code units when the fluctuating magnetic energy first reaches $80 \%$ of the average value of the fluctuating magnetic energy at the saturation stage.

while the magnetic energy densities do show strong dependence on $v(=\eta$ ) (see Cho \& Vishniac 2000a for more discussion for $B_{0} \approx 0$ limit). In the case of hyperviscosity simulations, the ratio $b^{2} / v^{2}$ for $B_{0} \rightarrow 0$ is greater than 0.6. When $B_{0}>0.1$, magnetic energy, $\left(B_{0}^{2}+b^{2}\right) / 2$, can be larger than kinetic energy, $v^{2} / 2$, for $256 \mathrm{H} 3$ runs.

Second, when the external fields are not very strong (say, $B_{0} \lesssim 0.2$ ), the fluctuating energy densities follow

$$
\begin{aligned}
& b^{2} \propto b^{(0) 2}+c_{b} v B_{0}, \\
& v^{2} \propto v^{(0) 2}-c_{v} v B_{0},
\end{aligned}
$$

where $c_{b}$ is almost independent of $v(=\eta)$ and $c_{v}$ weakly depends on $v(=\eta)$ and superscript ' $(0)$ ' denotes values for $B_{0}=0$. Equation (9) implies that

$$
b>\left(v B_{0}\right)^{1 / 2} \gg B_{0} .
$$

\subsection{Scaling of Total Energy}

One consequence of the above scaling relations is that the sum $v^{2}+\left(c_{v} / c_{b}\right) b^{2}$ is approximately independent of $B_{0}$ when the external fields are not very strong:

$$
v^{2}+\left(c_{v} / c_{b}\right) b^{2} \approx v^{(0) 2}+\left(c_{v} / c_{b}\right) b^{(0) 2} .
$$

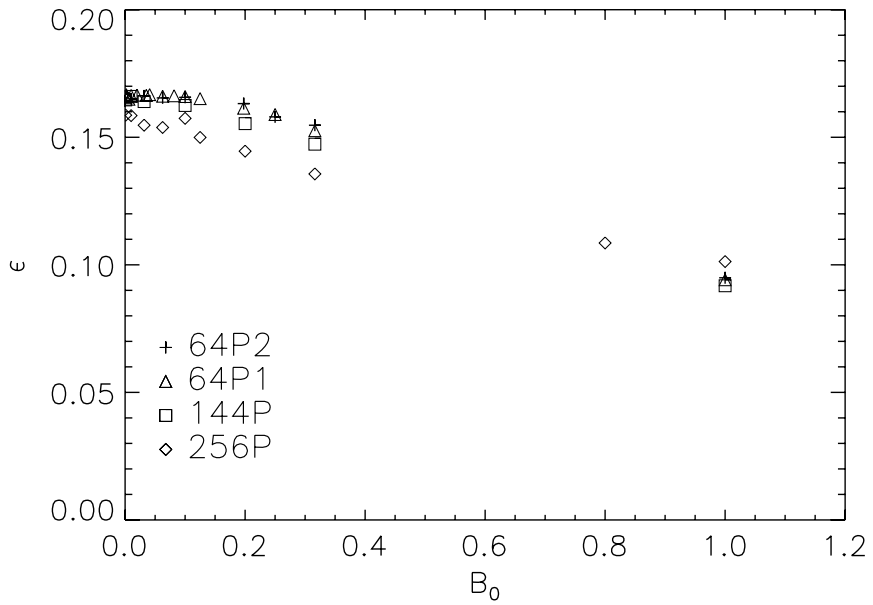

Figure 7. Average values of the total energy dissipation rate.

Figure 9 shows that total energy $\left(v^{2}+b^{2}\right)$ does not strongly depend on $B_{0}$. The constancy of the total energy is especially good for runs with high numerical resolutions $(256 \mathrm{P}$ and $256 \mathrm{H} 3$ runs). Therefore the figure implies that $\left(c_{v} / c_{b}\right) \approx 1$ for $256 \mathrm{P}$ and $256 \mathrm{H} 3$ runs. However, the value of $\left(c_{v} / c_{b}\right)$ is less than 1 for lower resolution runs. Since $v^{2}+b^{2}$ is virtually independent of $B_{0}$, we can use the quantity for normalization. For example, we may define the generalized large-scale eddy turnover time as $L /\left(v^{2}+b^{2}\right)^{1 / 2}$.

\subsection{Scaling of Energy Transfer Rate}

Cho and Vishniac (2000a) showed that, when the external fields are weak/zero, magnetic fields are amplified through field line stretching,

$$
D_{M} \propto(v-c B) B^{2} / L,
$$

where $D_{M}$ is magnetic dissipation and $c \approx 1 / 1$.6. Figure 10 confirms that this result is also true even when the external fields are strong. In the stationary state, the magnetic dissipation $\left(D_{M}\right)$ is balanced by the net energy transferred to the magnetic field from the velocity field. The right-hand side therefore tells us that the net energy transferred to the magnetic field is proportional to the large-scale eddy turnover rate $(v / L)$ minus an Alfvénic frequency $(B / L)$ times a constant. The large-scale eddy turnover rate is equal to the stretching rate of the magnetic field when the back reaction is zero. We identify the second term on the 

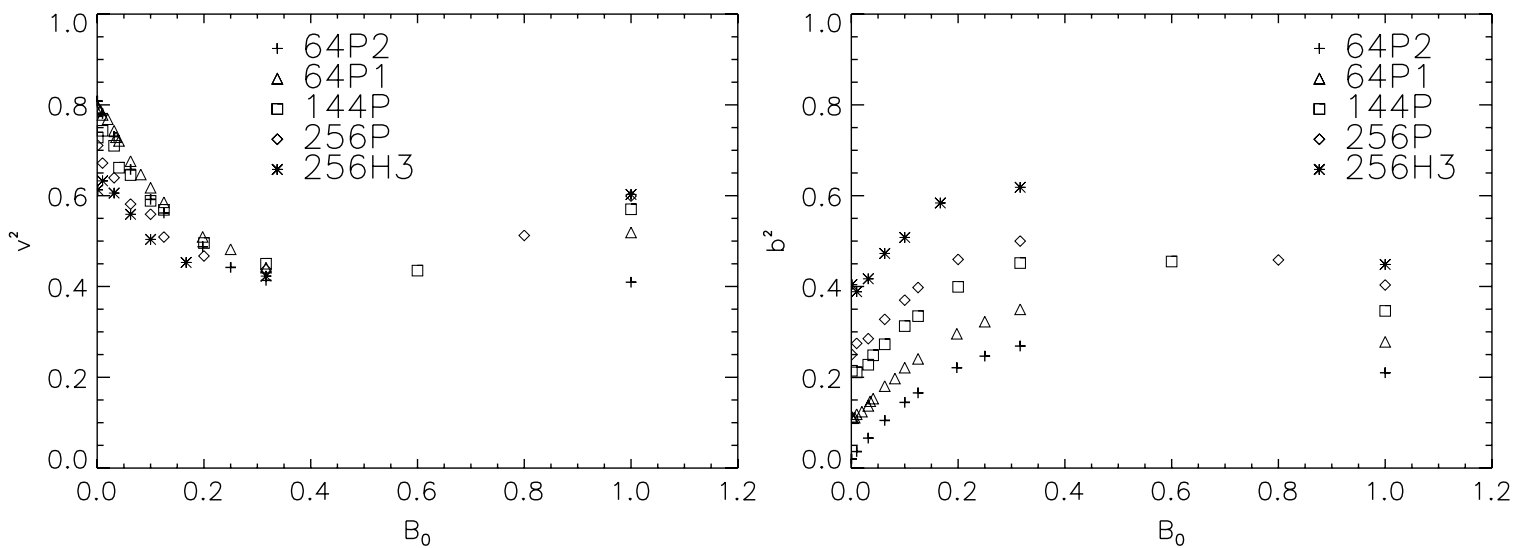

Figure 8. Average values of $v^{2}$ and $b^{2}$. Note that $b^{2}$ is the energy of fluctuating magnetic field. When $B_{0}>0.1$, magnetic energy, $\left(B_{0}^{2}+b^{2}\right) / 2$, can be larger than kinetic energy, $v^{2} / 2$, for $256 \mathrm{H} 3$ runs.

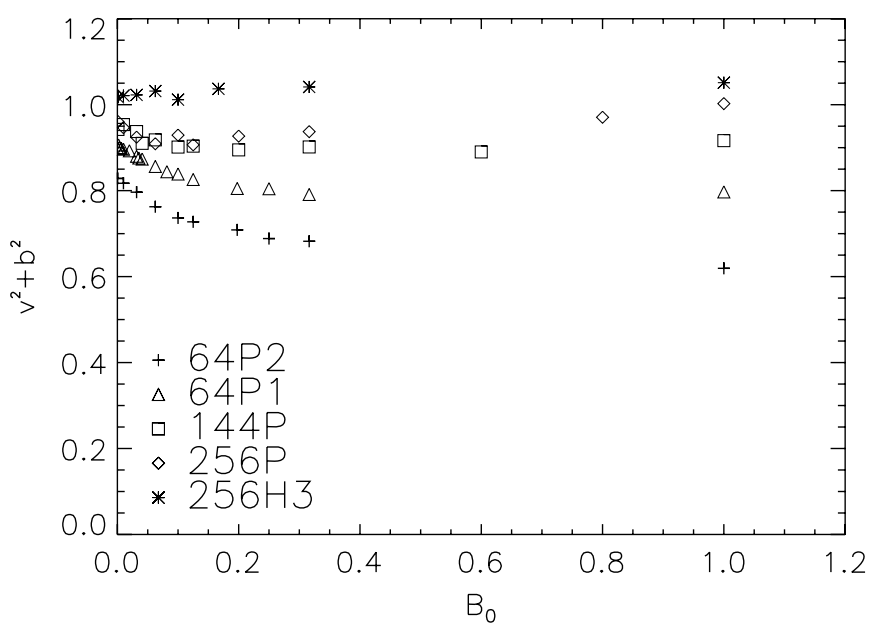

Figure 9. Total energy density and $B_{0}$. Total energy does not show strong dependence on the mean field strength.

right-hand side of this equation as the effect of the magnetic back reaction.

\subsection{Energy Spectra}

We plot the energy spectra in Figure 11. The kinetic spectrum peaks at $k \sim 2.5$, which is independent of $B_{0}$. The kinetic spectrum for the run with $B_{0}=0.1$ (thick solid line) has less energy at small $k$ s than that with $B_{0}=0.001$ (thick dashed line). On the other hand, the magnetic spectrum for $B_{0}=0.1$ (solid line) has more energy at small $k \mathrm{~s}$ than that for $B_{0}=0.001$ (dashed line). As the mean field strength grows, the peak of the magnetic spectrum moves from $\sim k_{L} / 2.5$ ( $B_{0} \approx 0$ case) to $\sim k_{L}\left(B_{0}=v_{\mathrm{rms}}\right.$ case), where $k_{L}$ is the wavenumber of the peak of kinetic energy spectra. Kinetic spectra are steeper than the Kolmogorov spectrum for small $k \mathrm{~s}$, while magnetic spectra are flatter. Therefore, one should be careful when using the Kolmogorov spectrum for MHD turbulence: the kinetic and magnetic spectra are not Kolmogorov for small $k$ 's when $B_{0}$ is weak. Since the slope of the total energy spectrum roughly follows Kolmogorov slope (see Figure 16 and Section 5), the slope of kinetic spectra at small $k \mathrm{~s}$ is a function of $B_{0}$ : when $B_{0}$ is small, kinetic spectra should be steeper than $k^{-5 / 3}$ and those for larger $B_{0}$ are closer to $k^{-5 / 3}$. When $B_{0} \sim v_{\text {rms }}$, the kinetic energy spectrum is very close to the Kolmogorov one consistent with the predictions in Goldreich \& Sridhar (1995)

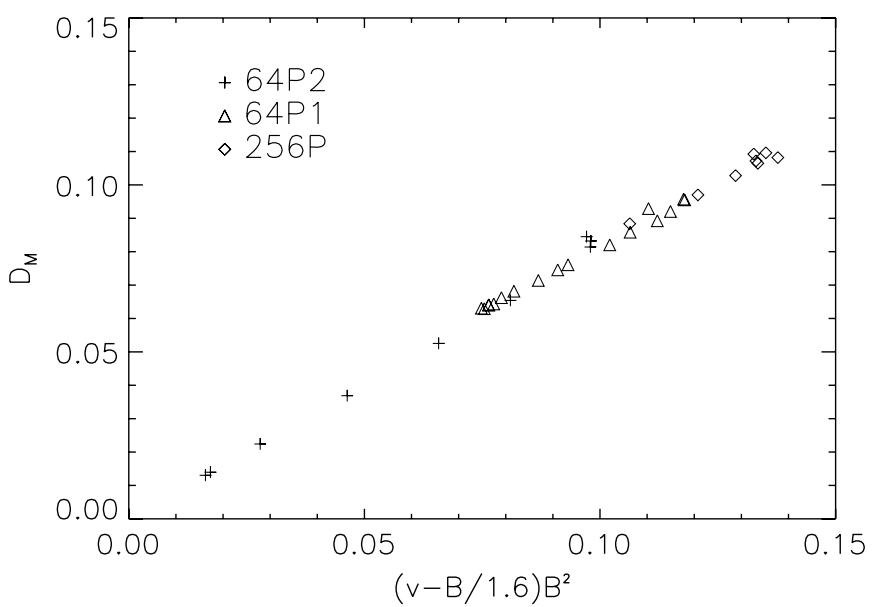

Figure 10. Stretching effect. Magnetic fields are amplified through field line stretching. The stretching rate is proportional to $v B^{2}$, which may be the stretching rate for passive vector fields, minus $B B^{2}$, which may be regarded as magnetic back reaction, times a constant. The value of $B_{0}$ ranges from 0 to $10^{-0.5}$. We use runs with physical viscosity only. Runs with $B_{0} \sim 1$, do not follow this relation.

as well as with some of the earlier simulations (Cho \& Vishniac 2000b; Cho et al. 2002). However, we cannot make a stronger conclusion with the present resolution (see Maron \& Goldreich 2001; Beresnyak \& Lazarian 2006; Mason et al. 2006).

\subsection{Anisotropy}

When $B_{0} \rightarrow v$, MHD turbulence tends to be anisotropic (Shebalin et al. 1983; Goldreich \& Sridhar 1995). Here we focus on global anisotropy of MHD turbulence with respect to the mean magnetic field $\mathbf{B}_{0} \cdot{ }^{11}$ Let $k_{\|}$be the wavenumber parallel to the mean field and $k_{\perp}$ the wavenumber perpendicular to the mean field. Matthaeus et al. (1998) showed that the anisotropy of MHD turbulence scales linearly with the ratio of perturbed and total magnetic strength $b / B$. Their result is mainly for $B_{0} \gtrsim v$. No systematic study is available for $B_{0}<v$ cases.

\footnotetext{
${ }^{11}$ In this subsection, we consider global anisotropy, not local anisotropy. That is, we calculate anisotropy with respect to the direction of the imposed field. When we calculate anisotropy with respect to directions that follow the local mean field in smaller subvolumes, we do obtain anisotropy for all values of $B_{0}$. This kind of anisotropy is local anisotropy. Scale-dependent anisotropy discussed in Goldreich \& Sridhar (1995) is local anisotropy (see Cho \& Vishniac 2000b and Cho et al. 2002 for details). Local anisotropy is important for local physical processes (e.g., pitch-angle scattering). But, what matters for external observers is mostly global anisotropy (or global isotropy).
} 


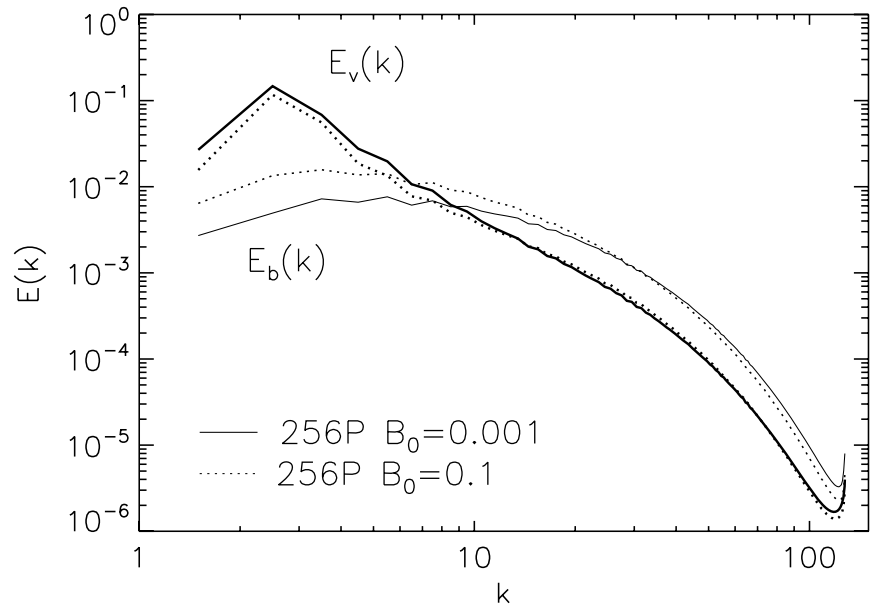

Figure 11. Energy spectra. The kinetic energy spectrum peaks at the driving scale (= energy injection scale). When the mean field gets stronger, the kinetic spectrum near the driving scale goes down. The magnetic energy spectrum peaks at wavelengths larger than the energy injection scale. The location of the peak moves toward smaller wavenumber as the mean field gets stronger. Note that, when $B_{0}<1$, the kinetic spectrum does not show a well defined power law and the average slope should be steeper than the Kolmogorov one near the energy injection scale.

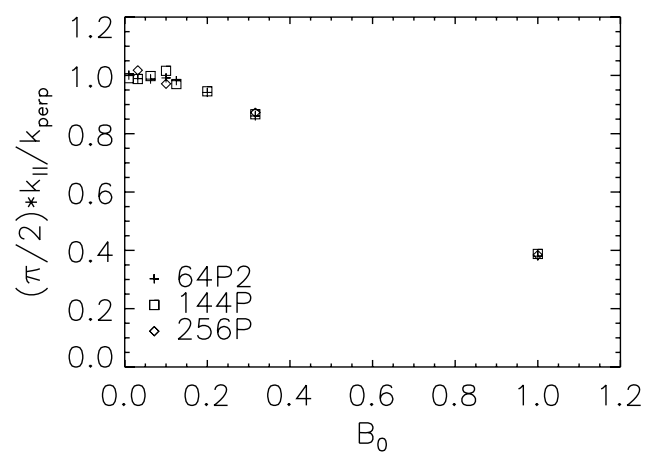

Figure 12. Global anisotropy. Here $k_{\|}$and $k_{\perp}$ are the average wavenumber along and perpendicular to the mean field direction, respectively. Turbulence remains almost isotropic when $B_{0} \lesssim 0.2$.

We study anisotropy of MHD turbulence in the $B_{0}<v$ limit by comparing average $k_{\|}$and $k_{\perp}$ :

$$
\frac{\left\langle k_{\|}\right\rangle}{\left\langle k_{\perp}\right\rangle}=\frac{\left\langle\int_{0}^{k_{\max }} d^{3} k k_{\|}\left|\hat{\mathbf{v}}_{\mathbf{k}}\right|^{2}\right\rangle_{t}}{\left\langle\int_{0}^{k_{\max }} d^{3} k k_{\perp}\left|\hat{\mathbf{v}}_{\mathbf{k}}\right|^{2}\right\rangle_{t}},
$$

where $k_{\max }$ is the maximum wavenumber and $\hat{\mathbf{v}}_{\mathbf{k}}$ is the Fourier component of velocity at $\mathbf{k}$. The result in Figure 12 shows that turbulence is virtually isotropic when $B_{0} \lesssim 0.2 v$. We note that there is no apparent anisotropy of order $B_{0} / v$. When $B_{0}$ becomes stronger, turbulence shows departure from isotropy. Note again that we focus on the cases of $B_{0} \leqslant v$ in this paper. It is also noteworthy that our result is valid when driving is isotropic.

\subsection{Kinetic Helicity}

In Figure 13, we plot normalized kinetic helicity, $H_{k} / v^{2}$, as a function of $B_{0}$. The kinetic helicity, $H_{k}=\langle\mathbf{v} \cdot \nabla \times \mathbf{v}\rangle$, does show suppression as $B_{0}$ increases. But, constancy of $H_{k} / v^{2}$ implies that the reduction in $H_{k}$ is due to the reduction in $v^{2}$. Therefore, the figure clearly shows that kinetic helicity is not suppressed strongly. Since we can write $H_{k}=\langle\mathbf{v} \cdot \nabla \times \mathbf{v}\rangle \sim k_{\text {peak, } H_{k}} v^{2}$, where $k_{\text {peak }, H_{k}}$ is the wavenumber at which kinetic helicity spectrum

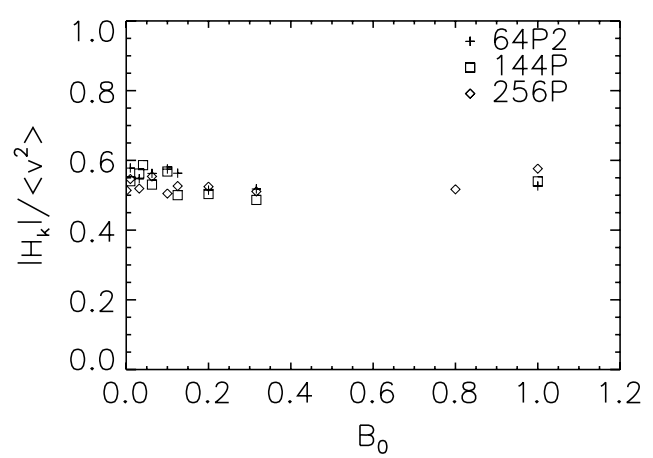

Figure 13. Normalized kinetic helicity $H_{K}$. This figure shows that kinetic helicity is not strongly suppressed.

peaks, the constancy of $H_{k} / v^{2}$ means the location of the peak wavenumber is not a function of $B_{0}$. Indeed the kinetic helicity spectra peak at the energy injection scale.

\subsection{Decay Timescale}

There have been a lot of studies on the decay law of MHD turbulence. The decay law and decay timescale of MHD turbulence are of great importance for the dynamics of molecular clouds and star formation.

Biskamp \& Müller (2000) discussed the decay of incompressible MHD turbulence in the limit of zero external field cases. However, there has been no systematic studies on the decay timescale for various values of the mean field strengths. The decay timescale of turbulence energy can be estimated by

$$
\left(v^{2}+b^{2}\right) / \epsilon .
$$

Figure 14 shows that the rate is not very sensitive to the strength of the mean field. Note that, even the case the Alfvén speed of the mean field $\left(B_{0}\right)$ is comparable to the rms velocity $(v \sim 1)$, the decay rate is not much reduced. This fact is consistent with earlier simulations of the decaying compressible MHD turbulence (Stone et al. 1998; Mac Low et al. 1998) as well as those of the incompressible one (Maron \& Goldreich 2001; Cho et al. 2002). We present a dimensionless dissipation coefficient

$$
D \equiv\left(\epsilon / 2 E_{\text {turb }}\right)\left(L^{\prime} / u^{\prime}\right)
$$

where $\epsilon$ is the energy dissipation rate, $E_{\text {turb }}=\left(v^{2}+b^{2}\right) / 2$, $3 u^{\prime 2}=v^{2}$, and

$$
L^{\prime}=\frac{\pi}{2 u^{\prime 2}} \int k^{-1} E_{v}(k) d k
$$

For 256P MHD runs the value of $L^{\prime}$ is around $\sim 0.76$ and weakly depends on $B_{0}$. For example, $L^{\prime}=0.76(256 \mathrm{P}-$ $B_{0} 10^{-3}$ and $\left.256 \mathrm{P}-B_{0} 10^{-1.5}\right), 0.72\left(256 \mathrm{P}-B_{0} 10^{-1}\right), 0.68(256 \mathrm{P}-$ $\left.B_{0} 10^{-0.5}\right)$, and $0.71\left(256 \mathrm{P}-B_{0} 1\right)$. The scale $L^{\prime}$ is a mathematical representation of the energy injection scale and the quantity $L^{\prime} / u^{\prime}$ is a kind of large-scale eddy turnover time. Since $L^{\prime} \sim$ $0.76, u^{\prime} \sim v / \sqrt{3}$, for 256P MHD runs, we have $L^{\prime} / u^{\prime} \sim 1.5$, which is smaller than $L / v$. In the $256 \mathrm{P}$ hydrodynamic run, $L^{\prime} \sim 0.62$. Note that the definition of the dimensionless dissipation rate is not exactly the same as that in Kaneda et al. (2003), in which they used

$$
\left(\epsilon / u^{\prime 2}\right)\left(L^{\prime} / u^{\prime}\right)
$$



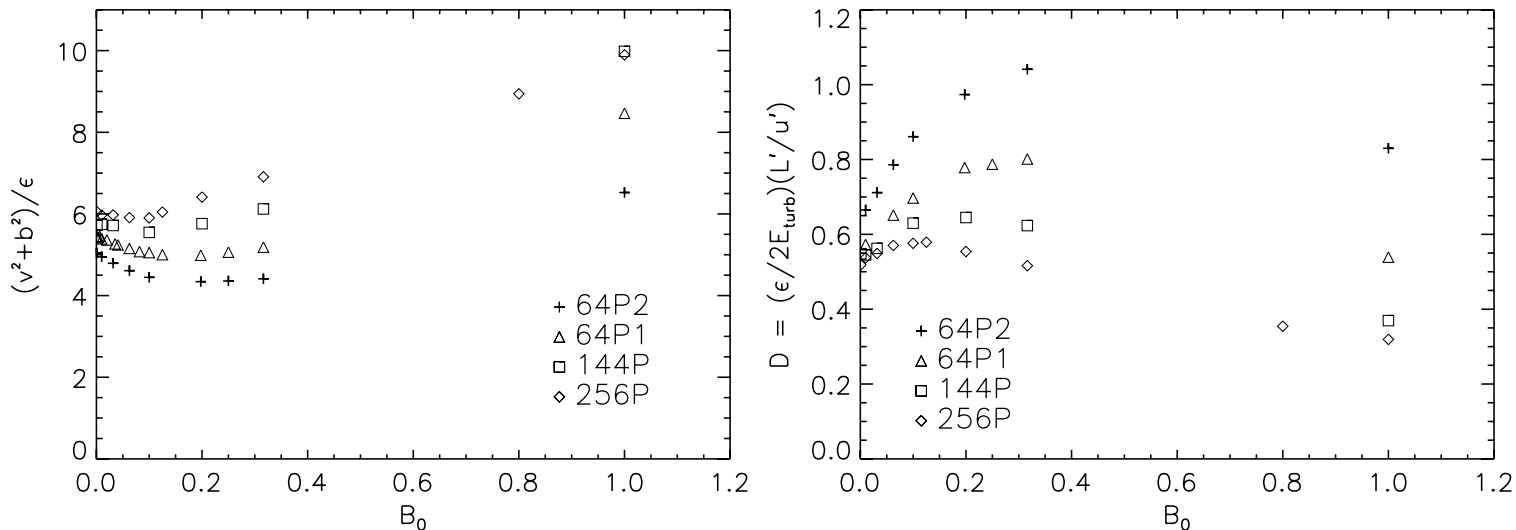

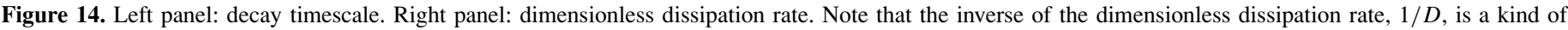
normalized turbulence decay time.
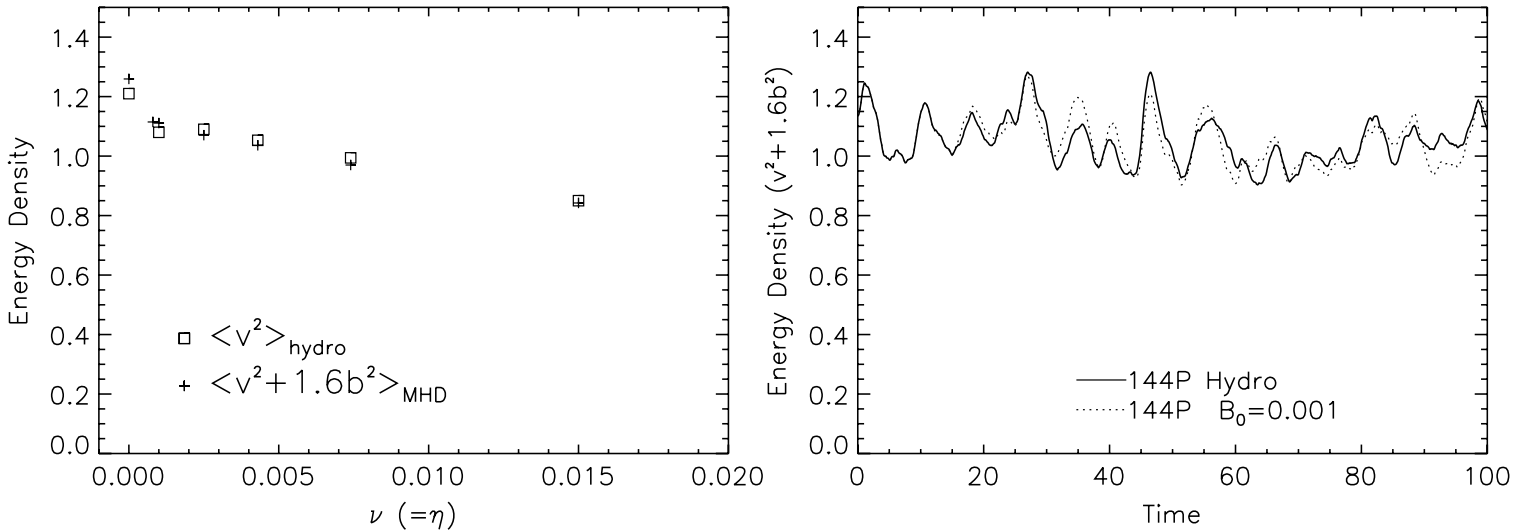

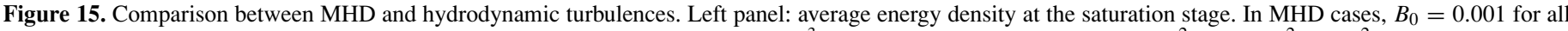

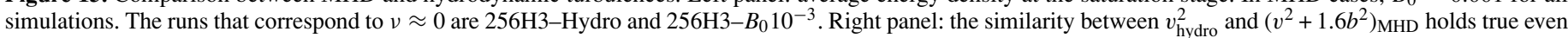
for time evolution.

The dimensionless dissipation coefficient is known to be around 0.5-0.6 both for hydrodynamic turbulence (Kaneda et al. 2003) and MHD turbulence (Stone et al. 1998; Haugen et al. 2004; see McKee \& Ostriker 2007). Our results are consistent with earlier findings: the value is around 0.5-0.6 when the mean field is weak. This value somewhat drops when the mean field is stronger. This has to do with the fact that the Kolmogorov constant depends on the value of the mean field (see the following section).

\section{COMPARISON WITH HYDRODYNAMIC SIMULATIONS}

It is often claimed that MHD turbulence with weak imposed magnetic field is similar to hydrodynamic turbulence. Is this true? When we introduce a mean field in a fully turbulent medium, what will happen to energy densities? In this section, we compare hydrodynamic and MHD turbulences.

We first compare energy densities. We run hydrodynamic and MHD simulations with the same initial conditions, except $B_{0}$, and the same prescribed driving force. Then we compare energy densities at the saturation stage. Figure 15 shows the behavior of the energy densities as a function of $v(=\eta)$. It is interesting that $\left\langle v^{2}\right\rangle$ in hydrodynamic turbulence and $\left\langle v^{2}+1.6 b^{2}\right\rangle$ in MHD cases scale similarly. The right panel of the figure shows that the similarity is also true for individual time basis.

The claim has a long history that total energy spectrum in MHD turbulence follows a Kolmogorov spectrum (see, for example, Kida et al. 1991; Müller \& Biskamp 2000). We plot the energy spectra of hydrodynamic and MHD turbulences in Figure 16. The compensated spectra in the right panel of the figure show that the total energy does show a slope compatible with the Kolmogorov one. However, the compensated spectrum of MHD turbulence can be higher or lower than that of hydrodynamic one depending on the mean field strength. In fact, the vertical location in the right panel of Figure 16 corresponds to the Kolmogorov constant $C_{K}$ :

$$
E(k)=C_{K} \epsilon^{2 / 3} k^{-5 / 3} \text {. }
$$

The Kolmogorov constant for hydrodynamic turbulence is around 1.6 (see, for example, Yeung \& Zhou 1997). On the other hand, in the MHD case, Biskamp \& Müller (2000) reported $C_{K} \sim 2.3$ in their decaying turbulence simulations. Our results show that the constant can be a function of $B_{0}$.

We compare the dimensionless dissipation coefficient (Equation (16)) for hydrodynamic and MHD turbulences in Figure 17. We define the coefficient as in Equation (16). For MHD cases we use $E_{\text {turb }}=\left(v^{2}+b^{2}\right) / 2$ and, for hydrodynamic cases, $E_{\text {turb }}=v^{2} / 2$. In the plot, we also mark the coefficient defined as in Equation (18), where $u^{\prime 2}$ is used instead of $2 E_{\text {turb }}$, for hydrodynamic cases (see squares in the figure). In hydrodynamic runs, the coefficient is dependent on the value of $v$. However, in MHD runs, the coefficient does not show strong dependence on $v(=\eta)$. 

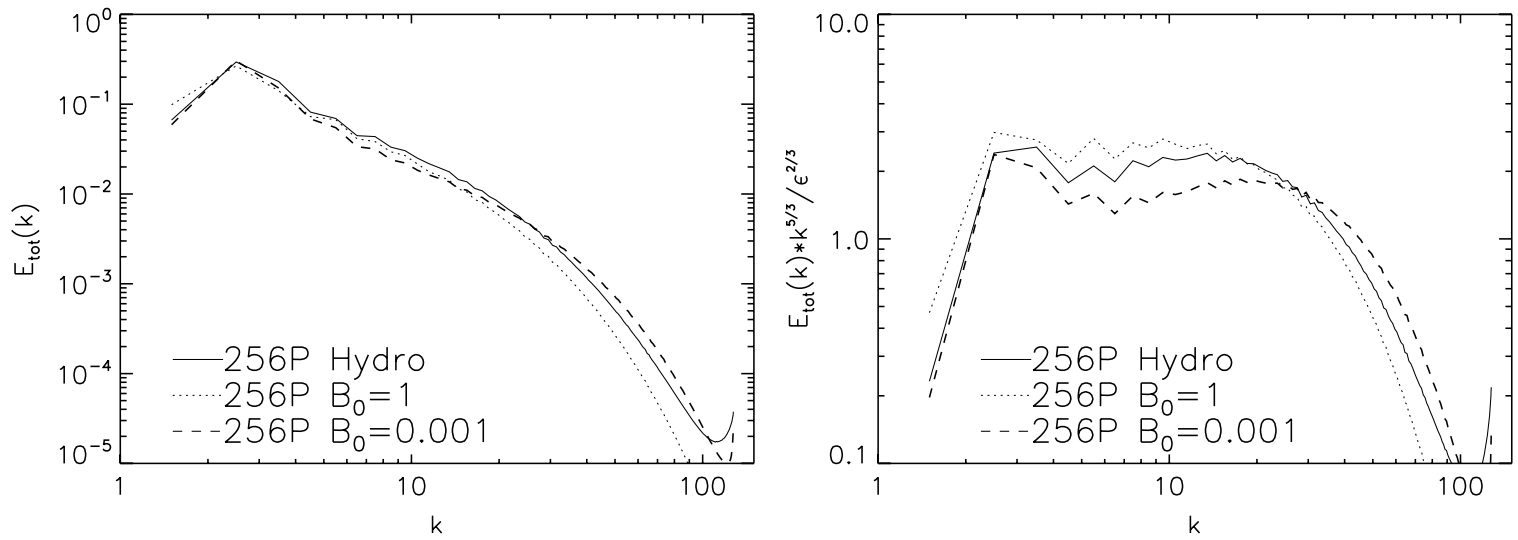

Figure 16. Comparison of energy spectra. Left panel: total energy spectra for MHD and kinetic energy spectrum for hydrodynamic turbulence. Right panel shows that the slope is compatible with Kolmogorov spectrum. Right panel: compensated spectra show that, when $B_{0}$ is weak, the total energy spectrum of MHD is lower than hydrodynamic one. This is understandable because $\left(v^{2}+b^{2}\right)_{\mathrm{MHD}}<\left(v^{2}+1.6 b^{2}\right)_{\mathrm{MHD}} \sim v_{\text {hydro }}^{2}$ regardless of the strength of the mean magnetic field and because $\epsilon \mathrm{S}$ in MHD with $B_{0} \approx 0$ and hydrodynamic cases are nearly same. However, when $B_{0} \sim v, \epsilon$ reduces and, therefore, the compensated spectrum is higher than the hydrodynamic one. We plot spectra averaged over time.

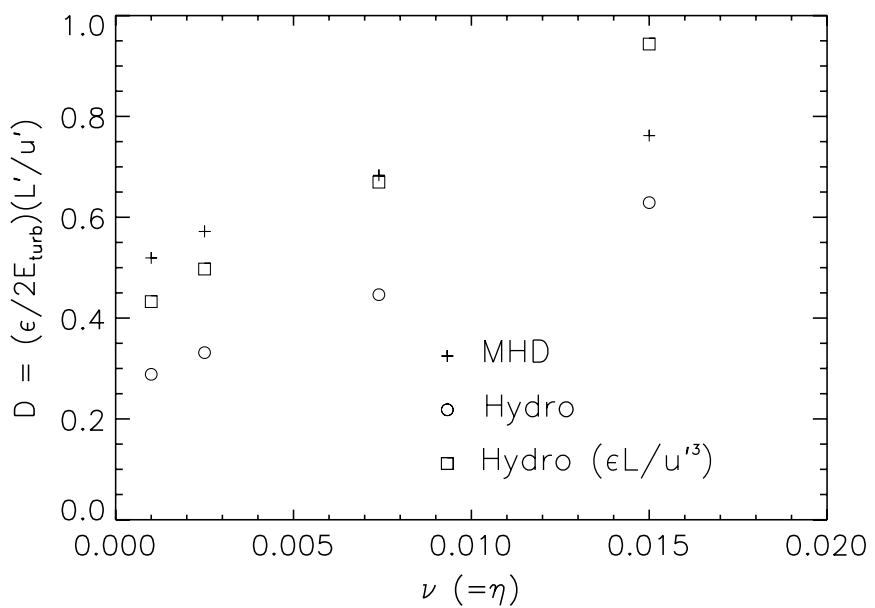

Figure 17. Dimensionless energy dissipation coefficient. Both hydrodynamic and MHD cases are shown. In MHD cases, $B_{0}=0.001$ for all simulations. See Equations (16) and (18) for the definition of the coefficient.

\section{DISCUSSION}

\subsection{Magnetic Reconnection}

Astrophysical magnetic fields are really ubiquitous. The origin of the large-scale regular magnetic fields is uncertain. Nevertheless, the turbulence dynamo discussed in this paper provides a simple and efficient way to produce small-scale random fields. It only requires the existence of turbulence, which is as ubiquitous as magnetic fields themselves (see Armstrong et al. 1995; Lazarian \& Pogosyan 2000; McKee \& Ostriker 2007). Nevertheless, small-scale turbulence dynamo assumes fast magnetic reconnection.

The reconnection is, indeed, fast in the numerical simulation that we deal with here. If, in astrophysical fluids, magnetic reconnection is slow, i.e., happens on timescales longer than the dynamical time, both the models of strong turbulence and the generation of magnetic field by turbulence are misrepresented by the numerics. In this situation we expect the formation of unresolved magnetic knots that should substantially alter the dynamics of the fluid and magnetic fields. If, however, reconnection if fast, i.e., happens on the timescales comparable to the Alfvénic wave propagation timescale, then our calculations do represent generation of astrophysical magnetic fields. We have ample astrophysical evidence and some models of fast re- connection are rather robust. For instance, a model of turbulent reconnection in Lazarian \& Vishniac (1999; see also Vishniac et al. 2003; Lazarian et al. 2004) predicts that magnetic field change its topology over the eddy turnover time. If, however, magnetic reconnection is not a robust process, i.e., is fast only in particular, e.g., collisionless environments (see Shay et al. 1999), the turbulent generation of magnetic field will differ substantially from one environment to another. Recent testing shows magnetic reconnection is indeed a fast process (Lazarian et al. 2008).

\subsection{Turbulence with Moderate Imposed Fields in Astrophysical Flows}

We have considered the effects of weak mean magnetic fields $\left(0 \leqslant B_{0} / \sqrt{4 \pi \rho} \leqslant v_{\text {rms }}\right)$ on MHD turbulence. Note that in our simulations $\left\langle v^{2}\right\rangle=v_{\mathrm{rms}}^{2} \sim 1$ and effectively we can assume $\rho=1$ for incompressible runs. In our code units, the factor $4 \pi$ disappears. Therefore, the parameter space we have considered is $0 \leqslant B_{0} \leqslant 1$. This work provides the missing link between two extreme limits: $B_{0} \rightarrow 0$ limit and $B_{0} / \sqrt{4 \pi \rho} \geqslant v_{\text {rms }}$ limit.

The limit of $B_{0} \rightarrow 0$ has been studied by many researchers (Pouquet \& Patterson 1978; Menegizzi et al. 1981; Kida et al. 1991; Cho \& Vishniac 2000a). In this paper, we have shown that there is no difference between turbulence with no external magnetic field and one with very weak external fields. In this sense, the limit of $B_{0} \rightarrow 0$ is not a special limit. Cho \& Vishniac (2000a) showed that energy equipartition between small-scale kinetic energy and total magnetic energy occurs at a scale about three times smaller than the energy injection scale. Magnetic spectrum of hyperviscosity simulation peaks at a wavelength about 2-3 times larger the kinetic energy peak. These results imply that, when we have a tangled magnetic field with no mean magnetic field in a fully developed turbulence, the characteristic size, $l_{B}$, of the magnetic field is about 2-3 times smaller than the energy injection scale of the turbulence.

When $0<B_{0} / \sqrt{4 \pi \rho}<v_{\text {rms }}$, the turbulence is called superAlfvénic in the interstellar medium community. The intracluster medium may fall in this regime (Ryu et al. 2008). Although it is still uncertain, the interstellar medium and/or molecular clouds in Galaxy may also fall in this regime (Padoan et al. 2004; Beck 2001). Beck (2001) gave an estimate of field strength derived from many different observations. He proposed that the regular component in our galaxy is $\sim 4 \mu \mathrm{G}$ and the total 
component $\sim 6 \mu \mathrm{G}$. On the other hand, Heiles \& Crutcher (2007) suggested that the energy ratio $E_{\text {turb }} / E_{\text {mag }}$ for cold neutral medium $(\mathrm{CNM})$ is $\sim 1.3$ in our galaxy. Figure 8 shows that, when $B_{0}>0.1, B^{2}$ can be larger than $v^{2}$ for $256 \mathrm{H} 3$ runs. Therefore, we expect that the mean field in the interstellar medium should be larger than $\sim 0.1$. Note that, when $B_{0}$ is larger than $\sim 0.2$, turbulence becomes globally anisotropic (Figure 12). Therefore, ISM turbulence is either marginally anisotropic if $B_{0} \sim 0.1$ or anisotropic if $B_{0}>0.2$. Turbulence in this case will reach saturation level very quickly within 3 or 4 eddy turnover times at most. The correlation length scale of magnetic field will be very close to the energy injection scale.

\subsection{Model for Growth of Magnetic Field in Astrophysical Flows}

In numerical simulations, the dissipation scale cannot be arbitrarily small. Instead, the dissipation scale is limited by the numerical resolution. In actual astrophysical fluids, the dissipation scale will be much smaller than that we can achieve with numerical simulations. Then what will be the time evolution of the magnetic energy in an actual astrophysical fluid which has a very high Reynolds number? Here, we only consider the case that $B_{0}$ is extremely small. When numerical resolution or Reynolds number is high, the dissipation scale is small and the eddy turnover time at the dissipation scale is short. As a result, the exponential growth is fast. Suppose that the linear growth stage begins at $t=t^{\prime}$. We plot the situation in the left panel of Figure 18. Time evolution of magnetic energy for $t>t^{\prime}$ is

$$
B^{2}(t)-v_{d}^{2}=C\left(t-t^{\prime}\right)
$$

where, $C$ is the slope at the linear growth stage. The kinetic energy at the dissipation scale, $\sim v_{d}^{2}$, and the time $t^{\prime}$ are given by

$$
v_{d}^{2} \sim v^{2}\left(l_{d} / L\right)^{2 / 3} \sim v^{2}(R e)^{-1 / 2},
$$

and

$$
\begin{array}{r}
t^{\prime} \sim \tau_{d} \ln \left(v_{d} / B_{0}\right)^{2} \\
\sim t_{\text {eddy }}\left(l_{d} / L\right)^{2 / 3} \ln \left(R e^{-1 / 4} v / B_{0}\right)^{2} \\
\sim t_{\text {eddy }}(R e)^{-1 / 2} \ln \left(R e^{-1 / 4} v / B_{0}\right)^{2}
\end{array}
$$

where $t_{\text {eddy }}$ is the large-scale eddy turnover time and we used the fact $l_{d} v_{d} / v \sim 1 \sim(L v / v)\left(l_{d} / L\right)\left(v_{d} / v\right) \sim(R e)\left(l_{d} / L\right)^{4 / 3}$. Here $l_{d}$ is the dissipation scale, $v$ is the large-scale rms velocity, $v_{d}$ is the velocity at the dissipation scale, $\tau_{d}$ is proportional to the eddy turnover time at the dissipation scale, and $R e=L v / v$ is the Reynolds number. Equations (21) and (22) imply that both $v_{d}^{2}$ and $t^{\prime}$ go to zero when the Reynolds number is very large. Therefore, we get

$$
B^{2}(t) \approx C t
$$

in real astrophysical fluids with very large Reynolds numbers When numerical resolution is finite, time evolution of $B^{2}$ will look like

$$
B^{2}(t) \approx C\left(t-t^{\prime}\right)
$$

because $v_{d}^{2}$ is negligible for reasonably high numerical resolutions.

Results in Section 3.4 (see left panel of Figure 3) show that the onset of the linear stage occurs later when the mean field is weak. Then how large is the delay? We plot the situation in the right panel of Figure 18. Note that the linear stage begins when energy equipartition is reached at the dissipation scale, which should be independent of the strength of the mean field. When
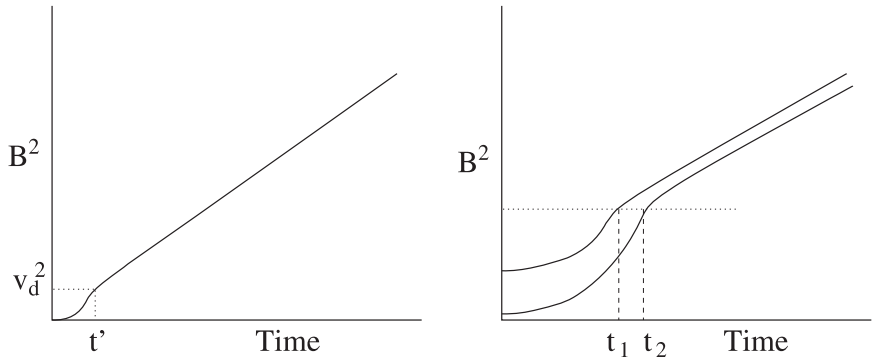

Figure 18. Growth of magnetic field energy in the presence of very weak mean magnetic field. Left panel: initially magnetic energy grows exponentially. Efolding time in this stage is the eddy turnover time at the dissipation scale. When the magnetic energy reaches equipartition with kinetic energy at the dissipation scale, exponential stage ends and linear growth stage begins. In actual astrophysical fluids with high Reynolds number, $t^{\prime}$ and $v_{d}^{2}$ are very small and, therefore, we expect $B^{2}(t) \propto t$ for most of the time until the saturation stage. Right panel: when the mean field strengths are different, the linear growth stage begins at different times. When the Reynolds number is high, $t_{2}-t_{1}$ will be very small.

the mean field is strong, the linear stage begins at $t_{1}$. When the mean field is weak, the linear stage begins later at $t_{2}$. Roughly speaking, the magnetic energy at the exponential growth stage is

$$
B^{2}(t) \propto B_{0}^{2} \exp \left(-t / \tau_{d}\right) .
$$

Therefore, we have

$$
B_{0,1}^{2} \exp \left(-t_{1} / \tau_{d}\right)=B_{0,2}^{2} \exp \left(-t_{2} / \tau_{d}\right) .
$$

Solving this equation, we get

$$
\begin{array}{r}
t_{2}-t_{1} \propto \tau_{d} \ln \left(B_{0,1} / B_{0,2}\right) \\
\propto t_{\text {eddy }}(R e)^{-1 / 2} \ln \left(B_{0,1} / B_{0,2}\right) .
\end{array}
$$

Of course, when the Reynolds number is large, $t_{2}-t_{1}$ is nearly zero, which means that the strength of the mean field does not matter much in astrophysical fluids.

\section{CONCLUSION}

We have studied the growth of magnetic field in the presence of weak/moderate mean magnetic fields. We have considered only the cases of $v=\eta$. We have found that equipartition between the kinetic and magnetic energy densities occurs at a scale somewhat smaller than the kinetic energy peak, which is consistent with previous results with lower numerical resolutions by Cho \& Vishniac (2000a). We have found that runs with different numerical resolutions and simulation parameters show consistent results for the slope of the linear growth stage. In real astrophysical flows with very weak mean field and very large Reynolds number, the growth of magnetic energy will follow

$$
\frac{B^{2}(t)}{2 E_{\text {turb }}} \sim 0.033 \frac{t}{L / \sqrt{v^{2}+B^{2}}} .
$$

We have also studied the effects of external magnetic fields on MHD turbulence. We have shown that magnetic energy density increases and kinetic energy density decreases as the external field becomes stronger. To be specific, we have shown that, when $B_{0} \leqslant 0.2$,

$$
\begin{gathered}
b^{2}-b^{(0) 2} \propto v B_{0}, \\
v^{2}-v^{(0) 2} \propto-v B_{0},
\end{gathered}
$$


where $b^{2}$ is the magnetic energy density in the presence of the external field and $b^{(0) 2}$ is the value when the external field is zero.

When the external magnetic field is not very strong $(\sim$ less than 0.2 times the rms velocity), the turbulence remains statistically isotropic, i.e., there is no apparent anisotropy of order $B_{0} / v$. The slope of kinetic spectra at small $k \mathrm{~s}$ is a function of $B_{0}$ : when $B_{0}$ is small, kinetic spectra should be steeper than $k^{-5 / 3}$ and those for larger $B_{0}$ are similar to $k^{-5 / 3}$.

We have compared MHD and hydrodynamic turbulences. Spectra of total energy in MHD turbulence are similar to those of hydrodynamic turbulence. The Kolmogorov constant in MHD cases can be higher or lower than that of hydrodynamic turbulence, depending on the strength of the mean field.

J.C. was supported by the Korea Research Foundation grant funded by the Korean Government (KRF-2006-331-C00136). A. B acknowledges support of the Ice Cube project. A. L. acknowledges support by NSF grants AST 0507164, as well as by the NSF Center for Magnetic Self-Organization in Laboratory and Astrophysical Plasmas. D. R. was supported by KOSEF through the grant of the Basic Research Program R01-2007000-20196-0. J. C. and D. R. were also supported by KICOS through the grant K20702020016-07E0200-01610 provided by MOST.

\section{REFERENCES}

Armstrong, J. M., Rickett, B. J., \& Spangler, S. R. 1995, ApJ, 443, 209

Batchelor, G. 1950, Proc. R. Soc. London A, 201, 405

Beck, R. 2001, Sp. Sci. Rev., 99, 243

Beresnyak, A., \& Lazarian, A. 2006, ApJ, 640, L175

Biskamp, D. 2003, Magnetohydrodynamic Turbulence (Cambridge: Cambridge Univ. Press)

Biskamp, D., \& Müller, W.-C. 2000, Phys. Plasmas, 7, 4889

Boldyrev, S. 2005, ApJ, 626, L37

Boldyrev, S. 2006, Phys. Rev. Lett., 96, 965002

Brandenburg, A., \& Subramanian, K. 2005, Phys. Reports, 417, 1

Brunetti, G., \& Lazarian, A. 2007, MNRAS, 378, 245

Cassano, R., \& Brunetti, G. 2005, MNRAS, 357, 1313

Chandran, B., \& Cowley, S. 1998, Phy. Rev. Lett., 80, 3077

Cho, J., \& Lazarian, A. 2002, Phys. Rev. Lett., 88, 5001

Cho, J., \& Lazarian, A. 2003, MNRAS, 345, 325

Cho, J., Lazarian, A., Honein, A., Knaepen, B., Kassinos, S., \& Moin, P. 2003, ApJ, 589, L77

Cho, J., Lazarian, A., \& Vishniac, E. T. 2002, ApJ, 564, 291

Cho, J., \& Vishniac, E. T. 2000a, ApJ, 538, 217

Cho, J., \& Vishniac, E. T. 2000b, ApJ, 539, 273

Galtier, S., Nazarenko, S. V., Newell, A. C., \& Pouquet, A. 2000, J. Plasma Phys., 63, 447

Goldreich, P., \& Sridhar, S. 1995, ApJ, 438, 763

Haugen, N., \& Brandenburg, A. 2004, Phys. Rev. E, 70, 036408
Haugen, N., Brandenburg, A., \& Dobler, W. 2003, ApJ, 597, L141

Haugen, N., Brandenburg, A., \& Dobler, W. 2004, Phys. Rev. E, 70, 016308

Heiles, C., \& Crutcher, R. 2007, arXiv:astro-ph/0501550

Higdon, J. 1986, ApJ, 309, 342

Iroshnikov, P. S. 1963, AZh, 40, 742

Kaneda, Y., Ishihara, T., Yokokawa, M., Itakuro, K., \& Uno, A. 2003, Phys. Fluids, 15, 21

Kazantsev, A. P. 1968, Soviet Phys.-JETP Lett., 26, 1031

Kida, S., Yanase, S., \& Mizushima, J. 1991, Phys. Fluids A, 3, 457

Kraichnan, R. H. 1965, Phys. Fluids, 8, 1385

Krause, F., \& Radler, K. 1980, Mean Field Magnetohydrodynamics and Dynamo Theory (Oxford: Pergamon Press)

Kulsrud, R., \& Anderson, S. 1992, ApJ, 396, 606

Kulsrud, R., Cen, R., Ostriker, J., \& Ryu, D. 1997, ApJ, 480, 481

Lazarian, A. 2006, ApJ, 645, L25

Lazarian, A., \& Beresnyak, A. 2006, MNRAS, 373, 1195

Lazarian, A., \& Pogosyan, D. 2000, ApJ, 537, 720

Lazarian, A., \& Vishniac, E. 1999, ApJ, 517, 700

Lazarian, A., Vishniac, E., \& Cho, J. 2004, ApJ, 603, 180

Lazarian, A., Vishniac, E., \& Kowal, G. 2008, arXiv:0812.4579

Lee, H., Ryu, D., Kim, J., Jones, T., \& Balsara, D. 2003, ApJ, 594, 627

Li, Z.-Y., \& Nakamura, F. 2004, ApJ, 609, L83

Mac Low, M.-M. 1999, ApJ, 524, 169

Mac Low, M.-M., Klessen, R., Burkert, A., \& Smith, M. 1998, Phys. Rev. Lett., 80,2754

Maron, J., \& Goldreich, P. 2001, ApJ, 554, 1175

Mason, J., Cattaneo, F., \& Boldyrev, S. 2006, Phys. Rev. Lett., 97, 975002

Matthaeus, W. H., Oughton, S., Ghosh, S., \& Hossain, M. 1998, Phy. Rev. Lett., 81,2056

McKee, C., \& Ostriker, E. 2007, ARA\&A, 45, 565

Menegizzi, M., Frisch, U., \& Pouquet, A. 1981, Phys. Rev. Lett., 47, 1060

Moffatt, H. K. 1978, Magnetic Field Generation in Electrically Conducting Fluids (Cambridge: Cambridge Univ. Press)

Müller, W.-C., \& Biskamp, D. 2000, Phys. Rev. Lett., 84, 475

Narayan, R., \& Medvedev, M. V. 2001, ApJ, 562, L129

Padoan, P., Jimenez, R., Juvela, M., \& Nordlund, A. 2004, ApJ, 604, L49

Padoan, P., \& Nordlund, A. 1999, ApJ, 526, 279

Parker, E. N. 1979, Cosmical Magnetic Fields (Oxford: Oxford Univ. Press)

Pouquet, A., \& Patterson, G. 1978, J. Fluid Mech., 85, 305

Ryu, D., Kang, H., Cho, J., \& Das, S. 2008, Science, 320, 909

Schekochihin, A., \& Cowley, S. 2007, in Magnetohydrodynamics - Historical Evolution and Trends, ed. S. Molokov, R. Moreau, \& H. Moffatt (Berlin: Springer) 85

Schekochihin, A., Iskakov, A., Cowley, S., McWilliams, J., Proctor, M., \& Yousef, T. 2007, New J. of Phys., 9, 300

Shebalin, J. V., Matthaeus, W. H., \& Montgomery, D. 1983, J. Plasma Phys., 29,525

Shay, M., Drake, J., Rogers, B., \& Denton, R. 1999, Geophys. Res. Lett., 26, 2163

Stone, J., Ostriker, E., \& Gammie, C. 1998, ApJ, 508, L99

Subramanian, K. 2008, arXiv:0802.2804

Tao, L., Cattaneo, F., \& Vainstein, S. I. 1993, in Theory of Solar and Planetary Dynamos, ed. M. R. E. Proctor, P. C. Matthews, \& A. M. Rucklidge (Cambridge: Cambridge Univ. Press), 303

Vazquez-semadeni, E., Kim, J., \& Ballesteros-Paredes, J. 2005, ApJ, 630, L49

Vishniac, E. T., Lazarian, A., \& Cho, J. 2003, in Turbulence and Magnetic Fields in Astrophysics, Lect. Notes in Phys., 614, ed. E. Falgarone \& T. Passot (Berlin: Springer) 376

Yeung, P., \& Zhou, Y. 1997, Phys. Rev. E, 56, 1746 